\title{
Invariants entiers en géométrie énumérative réelle
}

\author{
Jean-Yves Welschinger
}

30 octobre 2018

\begin{abstract}
Résumé
Je rappelle les divers problèmes de géométrie énumérative réelle desquels j'ai pu extraire des invariants à valeurs entières, fournissant un pendant réel aux invariants de Gromov-Witten. Je discute l'optimalité des bornes inférieures fournies par ces invariants ainsi que certaines de leurs propriétés arithmétiques. Je présente enfin davantage de résultats garantissant la présence ou l'absence de disques pseudoholomorphes à bord dans une sous-variété lagrangienne d'une variété symplectique donnée.
\end{abstract}

\section{Introduction}

Le nombre de racines complexes d'un polynôme générique à une variable de degré $d$ ne dépend pas du choix du polynôme et vaut $d$, tandis que lorsque ce polynôme est à coefficients réels, le nombre de ses racines réelles peut prendre toutes les valeurs de même parité que $d$ comprises entre 0 et $d$. Ceci tient au fait que le corps des nombres complexes est algébriquement clos au contraire du corps des nombres réels. Bien plus généralement, le nombre de solutions d'un «système de $n$ équations génériques »sur une variété projective complexe lisse de dimension $n$ ne dépend que du degré de ces équations, alors qu'il dépend fortement du choix, même générique, de ces équations lorsqu'elles sont à coefficients réels et considérées sur le lieu réel d'une variété algébrique réelle. (En fait d'équations, il conviendrait plutôt de parler de sections génériques de $n$ fibrés en droites holomorphes disons très amples). Chaque problème de géométrie énumérative réelle peut en principe s'interpréter de cette manière. La variété projective réelle est l'espace des modules des objets géométriques que l'on veut compter et les équations proviennent des conditions d'incidences que l'on impose à ces objets.

Le principal phénomène présenté dans cet article de synthèse est le suivant : il est parfois possible de compter ces objets géométriques réels en fonction d'un signe \pm de manière à extraire un entier indépendant du choix générique des conditions d'incidence. Dans le

Keywords : Enumerative geometry, rational curve, real algebraic variety, holomorphic discs. AMS Classification : 53D45, 14N35. 
premier paragraphe, nous observons ce phénomène en comptant les courbes $J$-holomorphes rationnelles réelles dans une variété symplectique réelle de dimension quatre en fixant leur classe d'homologie et leur imposant de passer par un nombre adéquat de points réels ou bien complexes conjugués. Nous utilisons en effet le langage de la géométrie symplectique pour étudier ces problèmes énumératifs, tenant compte des résultats de M. Gromov 9] selon lesquels le caractère algébrique des variétés ne joue aucun rôle dans ces problèmes énumératifs, seule l'ellipticité de l'opérateur de Cauchy-Riemann sous-jacent intervient. Les entiers que l'on extrait de ce problème énumératif fournissent un invariant par déformation des variétés symplectiques réelles de dimension quatre $\left(X, \omega, c_{X}\right)$, qui prend la forme d'une fonction $\chi: d \in H_{2}(X ; \mathbb{Z}) \mapsto \chi^{d}[T] \in \mathbb{Z}\left[T_{1}, \ldots, T_{N}\right]$ où $N$ désigne le nombre de composantes connexes du lieu réel $\mathbb{R} X$ de la variété. On définit des invariants analogues pour les variétés symplectiques « fortement semipositives $»$, par exemple positives, dans le troisième paragraphe et en incluant des conditions de tangence à une courbe réelle dans le deuxième. Ces derniers résultats s'appliquent en particulier à un problème classique de géométrie énumérative, le comptage des coniques tangentes à cinq coniques génériques données. Le nombre de solutions complexes vaut 3264, un résultat établi par de Joncquières au milieu du dix-neuvième siècle. On montre que le nombre de solutions réelles se trouve minoré par trente-deux lorsque les coniques réelles bordent cinq disques disjoints par exemple. En effet, la valeur absolue des invariants entiers que l'on introduit dans ce mémoire borne inférieurement le nombre de solutions réelles du problème énumératif que l'on considère.

Un deuxième phénomène apparaît dans cet article, l'optimalité de ces bornes inférieures. On montre en effet dans le premier paragraphe que dans le cas des variétés symplectiques réelles de dimension quatre, lorsque le lieu réel possède une sphère, un tore ou bien, sous des conditions plus restrictives, un plan projectif réel et lorsqu'au plus un point est choisi réel et dans cette composante, il existe une structure presque complexe générique $J$ pour laquelle le nombre de courbes $J$-holomorphes rationnelles réelles satisfaisant nos conditions d'incidence vaut exactement la valeur absolue de notre invariant, ceci quelle que soit la classe d'homologie de ces courbes rationnelles. Ce résultat vaut également pour la quadrique ellipsoïde de dimension trois, comme établi dans le troisième paragraphe. Cette optimalité est établie à l'aide de méthodes issues de la théorie symplectique des champs, méthodes qui nous permettent également parfois de calculer le signe de notre invariant, d'établir des congruences satisfaites par ce dernier ainsi que de fournir des formules le calculant dans certains cas, calculs que l'on mène explicitement en bas degrés. Tous ces résultats font l'objet du premier paragraphe de cet article. En utilisant la notion d'involution antibirationnelle sur une variété symplectique de dimension quatre, on montre de la même manière dans le quatrième paragraphe l'existence de disques $J$-holomorphes à bords dans le tore de Clifford et satisfaisant des conditions d'incidences ponctuelles. Dans le cas d'une sphère lagrangienne dans une variété symplectique négative ou nulle, on montre au contraire dans ce même paragraphe, pour tout $E>0$, l'existence de structures presque-complexes $J$ pour lesquelles aucun disque ou membrane $J$-holomorphe d'énergie inférieure à $E$ ne repose sur cette sphère, un résultat analogue à nos résultats d'optimalités puisqu'on atteint ainsi le minimum possible du nombre de disques ou membranes $J$-holomorphes. Remarquons à propos que l'obtention d'invariants entiers ou rationnels à partir du comptage des disques 
$J$-holomophes à bords dans une sous-variété lagrangienne est un problème classique de géométrie symplectique (et de la théorie des cordes ouvertes en physique théorique) pour lequel peu de solutions existent. Notre approche en fournit une lorsque la lagrangienne est fixée par une involution antiholomorphe. Remarquons également que l'absence de disques $J$-holomorphes pour certaines structures permet de définir l'homologie de Floer pour des sphères lagrangiennes dans les variétés symplectiques à première classe de Chern nulle, un autre problème classique de géométrie symplectique (et de symétrie miroir en physique théorique).

Le présent article est largement issu de mon mémoire d'habilitation à diriger des recherches, laquelle fut soutenue à l'École normale supérieure de Lyon en mars 2008.

\section{Remerciements :}

Je remercie le Centre national de la recherche scientifique ainsi que l'Agence nationale de la recherche pour leurs soutiens sans lesquels je n'aurais pu réaliser ces travaux.

\section{Invariants énumératifs des variétés symplectiques réelles de dimension quatre}

\subsection{Définition des invariants}

Soit $\left(X, \omega, c_{X}\right)$ une variété symplectique réelle fermée de dimension quatre, par quoi on entend une variété symplectique fermée de dimension quatre $(X, \omega)$ équipée d'une involution $c_{X}$ satisfaisant la relation $c_{X}^{*} \omega=-\omega$. Le lieu fixe $\mathbb{R} X$ de cette involution est supposé ici non-vide, c'est le lieu réel de la variété, lequel a la propriété d'être lagrangien. Ses composantes connexes sont étiquetées $(\mathbb{R} X)_{1}, \ldots,(\mathbb{R} X)_{N}$. On note $\mathcal{J}_{\omega}$ l'espace des structures presque-complexes $\omega$-positives de $(X, \omega)$ de classe $C^{l}, l \gg 1$ et $\mathbb{R} \mathcal{J}_{\omega} \subset \mathcal{J}_{\omega}$ le sous-espace des structures $J$ qui rendent l'involution $c_{X} J$-antiholomorphe. Ce sont tous deux des variétés de Banach séparables non-vides et contractiles.

Soit $d \in H_{2}(X ; \mathbb{Z})$ une classe d'homologie satisfaisant la relation $\left(c_{X}\right)_{*} d=-d$ et $J \in \mathbb{R} \mathcal{J}_{\omega}$ une structure presque-complexe générique. Les courbes J-holomorphes rationnelles réelles homologues à $d$, c'est-à-dire les sphères $J$-holomorphes invariantes par $c_{X}$ et homologues à $d$, forment alors un espace de dimension $c_{1}(X) d-1$, où $c_{1}(X)$ désigne la première classe de Chern de la variété $(X, \omega)$. Nous supposons cette dimension positive ou nulle, puisque le cas contraire signifie que l'espace en question est vide, puis faisons chuter cette dimension à zéro en imposant quelques contraintes à ces courbes, à savoir de passer par une collection $\underline{x}$ de $c_{1}(X) d-1$ points distincts. Ces derniers peuvent être choisis réels, c'est-à-dire fixés par $c_{X}$, ou bien complexes conjugués, c'est-à-dire échangés par $c_{X}$; nous noterons $r_{i}$ le nombre de points réels choisis dans $(\mathbb{R} X)_{i}, i \in\{1, \ldots, N\}$, et $r_{X}$ le nombre de paires de points complexes conjugués, de sorte que $2 r_{X}+\sum_{i=1}^{N} r_{i}=c_{1}(X) d-1$. L'ensemble $\mathcal{R}_{d}(\underline{x}, J)$ des courbes $J$-holomorphes rationnelles réelles homologues à $d$ qui satisfont ces contraintes supplémentaires est fini. Ces courbes sont de plus toutes irréductibles, immergées et n'ont que des points doubles transverses comme singularités. Remarquons 
que le cardinal $R_{d}(\underline{x}, J)=\# \mathcal{R}_{d}(\underline{x}, J)$ dépend en général des choix auxiliaires de la structure presque complexe et de la configuration de points, essentiellement parce que le corps des réels n'est pas algébriquement clos. Nous allons montrer qu'il en devient indépendant lorsque l'on compte ces courbes en fonction d'un signe convenablement choisi.

Soit $C \in \mathcal{R}_{d}(\underline{x}, J)$, le nombre total de points doubles de $C$ se calcule par la formule d'adjonction et vaut $\delta=\frac{1}{2}\left(d^{2}-c_{1}(X) d+2\right)$. Les points doubles réels de $C$ sont de deux natures différentes. Ils peuvent être l'intersection locale de deux branches réelles ou bien l'intersection locale de deux branches complexes conjuguées. Ces points doubles réels sont dits non-isolés dans le premier cas et isolés dans le second

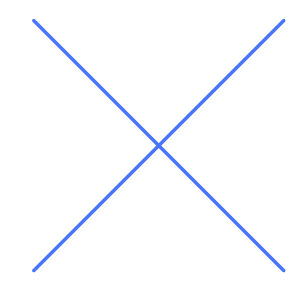

Point double réel non isolé

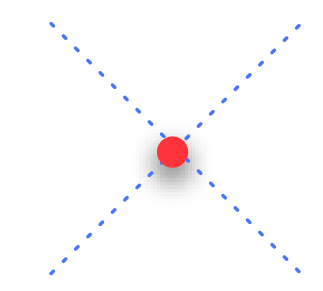

Point double réel isolé

Notons $m(C)$ le nombre de points doubles réels isolés de $C$, c'est la masse de $C$; elle est majorée par $\delta$. Pour tout entier $m$ compris entre 0 et $\delta$, on note $n_{d}(m)$ le nombre de courbes $C \in \mathcal{R}_{d}(\underline{x}, J)$ de masse $m$. Posons finalement

$$
\chi_{r}^{d}(\underline{x}, J)=\sum_{m=0}^{\delta}(-1)^{m} n_{d}(m),
$$

où $r=\left(r_{1}, \ldots, r_{N}\right)$.

Théorème 1.1 ([23], [25]) Soient $\left(X, \omega, c_{X}\right)$ une variété symplectique réelle fermée de dimension quatre, $N$ le nombre de composantes connexes de son lieu réel et $d \in H_{2}(X ; \mathbb{Z})$ satisfaisant $c_{1}(X) d>0$. Soient $\underline{x} \subset X$ une configuration réelle de $c_{1}(X) d-1$ points distincts et $r=\left(r_{1}, \ldots, r_{N}\right)$ le $N$-uplet associé. L'entier $\chi_{r}^{d}(\underline{x}, J)$ est indépendant du choix de $\underline{x}$ et du choix générique de $J \in \mathbb{R} \mathcal{J}_{\omega}$.

Le Théorème 1.1 permet de noter cet entier $\chi_{r}^{d}$ sans ambiguïté. Lorsque $\sum_{i=1}^{N} r_{i}$ n'a pas la même parité que $c_{1}(X) d-1$, on pose $\chi_{r}^{d}=0$. On note alors $\chi^{d}[T]$ la fonction génératrice $\sum_{|r|=0}^{c_{1}(X) d-1} \chi_{r}^{d} T^{r} \in \mathbb{Z}\left[T_{1}, \ldots, T_{N}\right]$, où $T^{r}=T_{1}^{r_{1}} \ldots T_{N}^{r_{N}}$ et $|r|=r_{1}+\cdots+r_{N}$. Cette fonction est de même parité que $c_{1}(X) d-1$ et tous ses monômes ne dépendent en fait que d'une indéterminée. En effet, la partie réelle d'une sphère holomorphe réelle étant connexe, l'invariant $\chi_{r}^{d}$ est contraint de s'annuler lorsque les points réels de $\underline{x}$ ne sont pas tous choisis dans une même composante $L$ du lieu réel. On adoptera la notation $\chi_{r}^{d}(L)$ pour indiquer que les $|r|$ points réels sont choisis dans $L$. On renvoie le lecteur à [25] pour une étude de la dépendance de $\chi_{r}^{d}$ en fonction de $r$. 
Ainsi, la fonction $\chi: d \in H_{2}(X ; \mathbb{Z}) \mapsto \chi^{d}[T] \in \mathbb{Z}\left[T_{1}, \ldots, T_{N}\right]$ ne dépend que de la variété symplectique réelle fermée de dimension quatre $\left(X, \omega, c_{X}\right)$ et est invariante par déformation de cette dernière. Ceci signifie que si $\omega_{t}$ est une famille continue de formes symplectiques satisfaisant $c_{X}^{*} \omega_{t}=-\omega_{t}$, alors la fonction $\chi$ est la même pour tous les triplets $\left(X, \omega_{t}, c_{X}\right)$. Existe-t-il des invariants énumératifs analogues à ceux qui ressortent du Theorème 1.1 en genre quelconque, en dimension quelconque et avec des conditions d'incidence quelconques? Nous n'avons que des débuts de réponses à ces questions.

\subsection{Bornes inférieures et optimalité}

Le nombre $R_{d}(\underline{x}, J)$ de courbes $J$-holomorphes rationnelles réelles homologues à $d$ qui contiennent l'ensemble $\underline{x}$ de points que l'on s'est donné se retrouve ainsi borné inférieurement par la valeur absolue de l'invariant $\chi_{r}^{d}$. Ce nombre est par ailleurs toujours majoré par le nombre total de courbes $J$-holomorphes rationnelles homologues à $d$ et contenant $\underline{x}$, lequel nombre $N_{d}$ ne dépend ni de $J$ générique, ni de $\underline{x}$; c'est un invariant de Gromov-Witten de genre zéro de la variété $(X, \omega)$. Ainsi,

Corollaire 1 ([25]) Sous les hypothèses du Théorème 1.1, l'encadrement

$$
\left|\chi_{r}^{d}\right| \leq R_{d}(\underline{x}, J) \leq N_{d}
$$

vaut pour tout choix de $\underline{x}$ et tout choix générique de $J \in \mathbb{R} \mathcal{J}_{\omega}$.

Les bornes inférieures apparaissant dans ce Corollaire 1 se trouvent être parfois optimales. C'est-à-dire qu'il est parfois possible d'exhiber une configuration $\underline{x}$ et une structure générique $J \in \mathbb{R} \mathcal{J}_{\omega}$ telles que toutes les courbes $J$-holomorphes rationnelles réelles comptées par $\chi_{r}^{d}$ le sont en fonction d'un unique et même signe. Nous présentons dans ce paragraphe les situations dans lesquelles nous avons été en mesure de montrer cette optimalité.

Théorème $1.2([29],[30])$ Soit $\left(X, \omega, c_{X}\right)$ une variété symplectique réelle fermée de dimension quatre et soit $d \in H_{2}(X ; \mathbb{Z})$ une classe d'homologie satisfaisant $\left(c_{X}\right)_{*} d=-d$. Supposons que le lieu réel de cette variété possède une sphère ou un plan projectif réel $L$. Dans ce dernier cas, supposons que $\left(X, \omega, c_{X}\right)$ est elle-même symplectomorphe au plan projectif complexe éclaté en six boules complexes conjuguées au maximum. Les bornes inférieures apparues dans le Corollaire 1 sont sous ces hypothèses optimales dès que $0 \leq$ $r \leq 1$. Le signe de l'invariant $\chi_{r}^{d}(L)$ est en outre dans ce cas déterminé par l'inégalité $(-1)^{\frac{1}{2}\left(d^{2}-c_{1}(X) d+2\right)} \chi_{r}^{d}(L) \geq 0$.

Remarque 1 La dernière partie du Théorème 1.2 signifie que le signe du coefficient de plus bas degré du polynôme $\chi^{d}(T)$ introduit au paragraphe 1.1 s'interprète comme la parité du genre lisse de la classe $d$. Le fait que ce signe puisse être négatif en degrés congrus à trois ou quatre modulo quatre dans le plan projectif complexe met en défaut la Conjecture 6 de [14]. 
Corollaire 2 ([30]) Soit $d$ une classe d'homologie de dimension deux du plan projectif complexe ou de la quadrique ellipsoïde et $0 \leq r \leq 1$. Les bornes inférieures (1) sont atteintes pour la structure complexe standard lorsque les points complexes conjugués sont choisis très proches d'une conique imaginaire pure dans le premier cas et d'une section hyperplane réelle disjointe de $L$ dans le second.

Théorème $1.3([30])$ Soit $\left(X, \omega, c_{X}\right)$ une variété symplectique réelle fermée de dimension quatre dont le lieu réel possède un tore $L$ et soit $d \in H_{2}(X ; \mathbb{Z})$ une classe d'homologie satisfaisant $\left(c_{X}\right)_{*} d=-d$. Les bornes inférieures du Corollaire 1 sont optimales lorsque $r=1$. Lorsque le lieu réel est connexe -réduit au tore $L$-, l'invariant $\chi_{1}^{d}(L)$ est en outre positif. Dans le cas général, le signe de l'invariant $\chi_{1}^{d}(L)$ est déterminé par l'inégalité $(-1)^{\frac{1}{2}\left(d^{2}-c_{1}(X) d+2\right)} \chi_{1}^{d}(L) \geq 0$ lorsque le lieu réel des courbes rationnelles ne s'annule pas dans $H_{1}(L ; \mathbb{Z} / 2 \mathbb{Z})$, tandis qu'il est déterminé par l'inégalité $(-1)^{\frac{1}{2}\left(d^{2}-c_{1}(X) d+2\right)} \chi_{1}^{d}(L) \leq 0$ lorsque ce dernier s'annule.

Remarque 2 Dans le cas particulier de la quadrique hyperboloïde, la positivité de $\chi_{1}^{d}(L)$ avait été observée dans [14] par d'autres méthodes.

De savoir si les bornes supérieures apparues dans le Corollaire 1 sont optimales est un problème classique de géométrie énumérative réelle pour lequel on ne sait presque rien. Le seule chose que je puisse signaler est le critère suivant.

Corollaire 3 ([25]) Sous les hypothèses du Théorème 1.1, supposons que $\chi_{r}^{d}$ est positif (resp. négatif). Supposons qu'il existe une configuration réelle de points $\underline{x}$ et une structure générique $J \in \mathbb{R} \mathcal{J}_{\omega}$ telles qu'il existe $\frac{1}{2}\left(N_{d}-\left|\chi_{r}^{d}\right|\right)$ courbes $J$-holomorphes rationnelles réelles de masses impaires (resp. paires) homologues à d et passant par $\underline{x}$. Alors, toutes les courbes J-holomorphes rationnelles homologues à $d$ et passant par $\underline{x}$ sont réelles, de sorte que les bornes supérieures du Corollaire 1 sont optimales.

Les bornes inférieures fournies par ces invariants sont-elles optimales en général? La question se pose déjà dans le cas du plan projectif (ou de l'espace projectif de dimension trois, voir le 3.1 .

\subsection{Congruences}

Étant donnée une classe d'homologie $d \in H_{2}(X ; \mathbb{Z})$ d'une variété symplectique réelle de dimension quatre $\left(X, \omega, c_{X}\right)$, nous noterons $g_{d}=\frac{1}{2}\left(d^{2}-c_{1}(X) d+2\right)$ le genre lisse de $d$ et $c_{d}=c_{1}(X) d-1$ le degré attendu du polynôme $\chi^{d}(T)$ défini au $\$ 1.1$.

Théorème $1.4([30])$ Soit $\left(X, \omega, c_{X}\right)$ une variété symplectique réelle fermée de dimension quatre dont le lieu réel possède une composante connexe $L$ homéomorphe à une sphère. Soient $d \in H_{2}(X ; \mathbb{Z})$ et $r \in \mathbb{N}$. Lorsque $2 r+1<c_{d}$, la puissance $2^{\frac{1}{2}\left(c_{d}-2 r-1\right)}$ divise $\chi_{r}^{d}(L)$.

\section{Exemple :}


Le Théorème 1.4 s'applique à l'ellipsoïde de dimension deux lorsque $d$ est un multiple positif, disons $\delta>0$, d'une section plane réelle. Dans ce cas, $c_{d}=4 \delta-1$ et $g_{d}=\delta^{2}-2 \delta+1=$ $\delta+1 \bmod (2)$. Par conséquent, $2^{2 \delta-r-1}$ divise $\chi_{r}^{d}(L)$ lorsque $r<2 \delta-1$. Nous avons également montré dans [30] que $2^{2 \delta-r}$ divise $\chi_{r}^{d}(L)$ lorsque de plus $r=2 \delta+1 \bmod (4)$ ainsi que la congruence $\chi_{2 \delta-3}^{d}(L)=0 \bmod (16)$.

Théorème 1.5 ([30]) Soit $\left(X, \omega, c_{X}\right)$ une variété symplectomorphe au plan projectif complexe éclaté en six boules complexes conjuguées au maximum. Soit $d \in H_{2}(X ; \mathbb{Z})$ une classe satisfaisant $c_{d}=c_{1}(X) d-1 \geq 0$ et soient $r, r_{X}$ des entiers naturels satisfaisant la relation $r+2 r_{X}=c_{d}$. Lorsque $r+1<r_{X}$, la puissance $2^{r_{X}-r-1}$ divise $\chi_{r}^{d}(L)$.

\section{Exemple :}

Le Théorème 1.5 s'applique au plan projectif complexe où $d$ est un multiple positif, disons $\delta>0$, d'une droite complexe. Dans ce cas, $8^{\frac{1}{2}(\delta-r-1)}$ divise $\chi_{r}^{d}$ lorsque $r+1<\delta$. Nous avons également montré dans [30] que $2^{\frac{1}{2}(3 \delta-3 r-1)}$ divise $\chi_{r}^{d}$ lorsque de plus $r=\delta+1$ $\bmod (4)$ et $\chi_{\delta-3}^{d}=0 \bmod (64)$.

\subsection{Calculs}

L'invariant $\chi_{r}^{d}$ qui ressort du Theorème 1.1 fut rapidement estimé après que je l'ai introduit. G. Mikhalkin a proposé dans [16] un algorithme permettant, dans le cas des surfaces toriques réelles, le calcul de cet invariant lorsque le nombre $r$ de points choisis réels est maximal. Cet algorithme a été plus tard étendu par E. Shustin [20] pour un choix quelconque de points réels. Il a été utilisé par I. Itenberg, V. Kharlamov et E. Shustin [12] pour estimer cet invariant, fournissant notamment la minoration $\chi_{3 d-1}^{d} \geq \frac{1}{2} d$ ! dans le cas du plan projectif, le calcul en degré inférieur ou égal à cinq, puis l'asymptotique $\log \left|\chi_{c_{1}(X) d-1}^{d}\right| \cong \log N_{d}$ dans le cas des surfaces de Del Pezzo réelles $X$, voir [14]. Ces derniers ont également plus récemment obtenu une formule de type Caporaso-Harris tropicale [13. pour le calcul de $\chi_{3 d-1}^{d}$ dans le plan, après que A. Gathmann et H. Markwig [8] ont obtenus cette formule pour le calcul tropical de $N_{d}$. J. Solomon a également annoncé une formule calculant ces invariants $\chi_{r}^{d}$ dans le plan. E. Shustin [21] a adapté ces méthodes tropicales pour obtenir des résultats analogues dans le cas de la quadrique ellipsoïde. Les méthodes de théorie symplectique des champs que j'ai pour ma part utilisé ([29], [30]) m'ont également permis d'obtenir des formules de type Caporaso-Harris mais avec des conditions de tangence imaginaires conjuguées. Les invariants relatifs qui interviennent dans ces formules sont introduits au 2.1 .1 . Je ne rappelle pas ici les formules générales qui se trouvent dans [30], mais simplement quelques calculs explicites qui en découlent facilement.

Corollaire 4 ([30]) Soit $\left(X, \omega, c_{X}\right)$ une variété symplectomorphe au plan projectif complexe. Alors, $\chi^{4}(T)=o\left(T^{2}\right), \chi^{5}(T)=64+64 T^{2}+o\left(T^{3}\right), \chi^{6}(T)=1024 T+1536 T^{3}+o\left(T^{4}\right)$, $\chi^{7}(T)=-14336+11776 T^{2}+o\left(T^{3}\right)$ et $\chi^{8}(T)=-280576 T+o\left(T^{2}\right)$.

Remarquons que $\chi^{3}(T)=2 T^{2}+4 T^{4}+8 T^{8}$; ce calcul de $\chi^{d}(T)$ en degré trois et les phénomènes discutés ici s'obtiennent simplement en éclatant les neuf points base d'un pin- 
ceau de cubiques planes et en calculant la caractéristique d'Euler du lieu réel de la surface obtenue, comme observé par V. Kharlamov [15] déjà dans les années 90. Toutefois, même l'existence d'une quartique rationnelle réelle plane passant par onze points réels en position générale n'était pas connue avant l'introduction de ces invariants $\chi_{r}^{d}$. Les valeurs explicites de $\chi_{r}^{d}$ pour $d \leq 9$ et tout $r$ furent entre temps obtenues dans [1] comme conséquence d'une formule de type Caporaso-Harris tropicale. Ces résultats mirent en défaut la conjecture de monotonie de [14], de sorte que la fonction $r \mapsto \chi_{r}^{d}$ n'est en général ni positive, ni monotone.

Corollaire 5 ([30]) Soit $\left(X, \omega, c_{X}\right)$ une variété symplectomorphe à la quadrique ellipsoïde de dimension deux. On note h la classe d'une section plane réelle de bidegré $(1,1)$. Alors, $\chi^{2 h}(T)=2 T^{3}+4 T^{5}+6 T^{7}, \chi^{3 h}(T)=16 T+16 T^{2}+o\left(T^{3}\right), \chi^{4 h}(T)=-256 T+320 T^{3}+o\left(T^{4}\right)$ et $\chi^{5 h}(T)=26880 T+o\left(T^{2}\right)$.

Remarque 3 Cet invariant $\chi_{r}^{d}$ peut se définir purement en termes de fractions rationnelles complexes. Lorsque $r=4 d-1$ par exemple, il compte algébriquement le nombre de fractions rationnelles $u=P / Q, P, Q \in \mathbb{C}[X]$ de degrés $d$, modulo reparamétrage par les homographies réelles de $P G L_{2}(\mathbb{R})$, telles que l'image $u\left(\mathbb{R} P^{1}\right)$ interpole un ensemble donné générique de $4 d-1$ points de la sphère de Riemann. Le signe en fonction duquel il convient de compter ces fractions rationnelles $u$ est pair si u possède un nombre pair de points critiques dans chaque hémisphère $\mathbb{C} P^{1} \backslash \mathbb{R} P^{1}$ et impair sinon. Il serait intéressant d'étudier cet invariant de la quadrique ellipsoïde en travaillant uniquement avec des fractions rationnelles.

Quelle est l'asymptotique de l'invariant $\chi_{r}^{d}, r \leq 1$, calculé ici? Nos formules calculent l'invariant en fonction d'une somme sur des arbres décorés. Quels sont les arbres qui sont asymptotiquement dominants/négligeables? De plus, dans le cas du plan projectif par exemple, lorsque $r=3 d-1$, notre formule calcule l'invariant comme une somme sur des arbres dont certains contribuent positivement et d'autres négativement. Ceci garantit l'existence de structures presque-complexes pour lesquelles davantage de courbes rationnelles réelles satisfont nos conditions d'incidences que le nombre imposé par l'invariant $\chi_{3 d-1}^{d}$. Combien de courbes réelles a-t-on ainsi construit? Enfin, notre méthode de calcul suivie dans la première section s'applique à toute variété symplectique de dimension quatre et calcule l'invariant $\chi$ en fonction d'invariants de Gromov-Witten de surfaces rationnelles relatifs à des courbes de carré -2 ou -4 lorsque $L$ est une sphère ou un plan projectif réel. Que sait-on de ces invariants et qu'en déduire pour l'invariant $\chi$ ? Cette direction de recherche reste à développer. Par ailleurs, j'ignore dans quelles situations exactement il est possible de calculer l'invariant $\chi_{r}^{d}$ en fonction d'invariants relatifs imaginaires.

\section{Invariants relatifs des variétés symplectiques réelles de dimension quatre}

Les invariants $\chi_{r}^{d}$ introduits au $\$ 1.1$ sont définis par un comptage de courbes $J$-holomophes rationnelles réelles soumises à des conditions d'incidence ponctuelles. Ils forment ainsi un 
analogue réel aux invariants de Gromov-Witten de genre zéro ponctuels. J'ai également défini de tels invariants en admettant que les courbes soient soumises à des conditions de tangence avec une courbe donnée, dans l'esprit de la théorie des invariants relatifs. Ces conditions de tangence peuvent être réelles ou bien complexe conjuguées. Dans le cas de conditions réelles, je n'ai pu définir de tels invariants relatifs qu'en admettant une seule condition de tangence et encore m'a-t-il fallu faire intervenir plusieurs types de courbes singulières. J'expose ces résultats dans le \$2.1.1. J'ai pu en déduire des bornes inférieures pour le nombre de coniques réelles tangentes à cinq coniques données, un problème classique de géométrie énumérative. Dans le cas de conditions de tangence complexes conjuguées, la situation est bien meilleure et de tels invariants peuvent s'obtenir avec les mêmes méthodes que celles utilisées au 1.1 . Je n'ai en fait introduit et utilisé ces invariants que dans des cas très particuliers, en utilisant le langage de la théorie symplectique des champs. Ils m'ont été utiles pour mener les calculs présentés au $\$ 1.4$. J'expose ces résultats dans le $\$ 2.2$.

\subsection{Invariants relatifs réels}

\subsubsection{Définition des invariants}

Soient $\left(X, \omega, c_{X}\right)$ une variété symplectique réelle de dimension quatre, $d \in H_{2}(X ; \mathbb{Z})$ une classe d'homologie telle que $c_{1}(X) d \geq 2$ et $\underline{x}$ une configuration réelle de $c_{1}(X) d-2$ points distincts. Comme au $\$ 1.1$, on note $\mathbb{R} X_{1}, \ldots, \mathbb{R} X_{N}$ les composantes connexes de $\mathbb{R} X$ et $r_{i}$ le cardinal de $\underline{x} \cap \mathbb{R} X_{i}, i \in\{1, \ldots, N\}$. Soit $B \subset \mathbb{R} X$ une surface à bord lisse. En chaque point réel $x_{i}$ de $\underline{x}$, on choisit une droite vectorielle $T_{i}$ dans le plan tangent $T_{x_{i}} \mathbb{R} X$. Pour toute structure presque complexe $J \in \mathbb{R} \mathcal{J}_{\omega}$ suffisamment générique, on définit l'entier $\Gamma_{r}^{d, B}(J, \underline{x})$ comme la somme des nombres de courbes $J$-holomorphes rationnelles réelles qui réalisent la classe d'homologie $d$, passent par la configuration $\underline{x}$ et qui proviennent des quatre familles suivantes :

- Les courbes tangentes au bord de $B$, elles sont comptées en fonction de leurs masses et de leur contact intérieur ou extérieur à $B$ au point de tangence.

- Les courbes non-immergées, qui sont comptées en fonction de leurs masses et de la position du point de rebroussement par rapport à $B$.

- Les courbes possédant une des droites $T_{i}$ comme tangente, qui sont comptées en fonction de leurs masses et de la position du point $x_{i}$ correspondant à $T_{i}$ par rapport à $B$.

- Les courbes réductibles, qui sont comptées en fonction de leurs masses et d'une multiplicité qui est le nombre de points réels d'intersection entre les deux composantes irréductibles de la courbe, chacun de ces points devant être compté positivement ou négativement selon qu'il est intérieur ou extérieur à $B$.

Ainsi, en notant respectivement $\mathcal{T}_{a n}^{d}(J, \underline{x}), \mathcal{C} u s p^{d}(J, \underline{x}), \mathcal{T} a n^{d}(J, \underline{x})$ et $\mathcal{R}_{e} d^{d}(J, \underline{x})$ ces quatre ensembles finis de courbes $J$-holomorphes, l'entier $\Gamma_{r}^{d, B}(J, \underline{x})$ s'écrit 


$$
\sum_{C \in \cup \mathcal{T}_{\operatorname{Tan}}^{d}(J, \underline{x}) \cup \mathcal{T}_{a n^{d}}(J, \underline{x}) \cup \mathcal{C} u s p^{d}(J, \underline{x})}(-1)^{m(C)}\langle C, B\rangle-\sum_{C \in \mathcal{R e d}^{d}(J, \underline{x})}(-1)^{m(C)} \operatorname{mult}_{B}(C) .
$$

Dans cette somme, l'indice de contact $\langle C, B\rangle$ vaut -1 (resp. +1$)$ si $C \in \mathcal{T}_{a n}^{d}(J, \underline{x})$ et $\mathbb{R} C$ se trouve localement incluse dans (resp. en dehors de) $B$ au voisinage du point de tangence $y$ avec $\partial B$. Si $C \in \mathcal{C} u s p^{d}(J, \underline{x})$ (resp. $C \in \mathcal{T} a n^{d}(J, \underline{x})$ ), le point de rebroussement (resp. la droite $T_{i}, i \in I$ ) est unique et l'indice de contact $\langle C, B\rangle$ vaut -1 si ce point se situe en-dehors de $B$ et +1 sinon. Si $C$ est réductible, elle n'a que deux composantes irréductibles $C_{1}, C_{2}$, toutes deux réelles et

$$
\operatorname{mult}_{B}(C)=\sum_{y \in \mathbb{R} C_{1} \cap \mathbb{R} C_{2}}\langle y, B\rangle,
$$

où $\langle y, B\rangle$ vaut -1 lorsque $y$ est extérieur à $B$ et +1 s'il est intérieur.

Théorème 2.1 ([28]) Soient $\left(X, \omega, c_{X}\right)$ une variété symplectique réelle fermée de dimension quatre et $B \subset \mathbb{R} X$ une surface à bord lisse. Soient $N$ le nombre de composantes connexes de $\mathbb{R} X$ et $d \in H_{2}(X ; \mathbb{Z})$ satisfaisant $c_{1}(X) d>1, c_{1}(X) d \neq 4$. Soient $\underline{x} \subset X \backslash \partial B$ une configuration réelle de $c_{1}(X) d-2$ points distincts et $r=\left(r_{1}, \ldots, r_{N}\right)$ le $N$-uplet associé, supposé non nul. L'entier $\Gamma_{r}^{d, B}(J, \underline{x})$ est indépendant du choix de $\underline{x}$ et du choix générique de $J \in \mathbb{R} \mathcal{J}_{\omega}$.

Le Théorème 2.1 permet sans ambiguïté de noter $\Gamma_{r}^{d, B}$ cet entier. Lorsque $\sum_{i=1}^{N} r_{i}$ n'a pas la même parité que $c_{1}(X) d$, on pose $\Gamma_{r}^{d, B}=0$. Comme au $\$ 1.1$, on note alors $\Gamma^{d, B}[T]$ la fonction génératrice $\sum_{|r|=0}^{c_{1}(X) d-2} \Gamma_{r}^{d, B} T^{r} \in \mathbb{Z}\left[T_{1}, \ldots, T_{N}\right]$. Cette fonction est de même parité que $c_{1}(X) d$ et tous ses monômes ne dépendent en fait que d'une indéterminée.

Ainsi, la fonction $\Gamma^{B}: d \in H_{2}(X ; \mathbb{Z}) \mapsto \Gamma^{d, B}[T] \in \mathbb{Z}\left[T_{1}, \ldots, T_{N}\right]$ ne dépend que du quadruplet $\left(X, \omega, c_{X}, B\right)$. Elle est en outre invariante par déformation de ce quadruplet au sens où si $\omega_{t}$ est une famille continue de formes symplectiques satisfaisant $c_{X}^{*} \omega_{t}=-\omega_{t}$ et $B_{t} \subset \mathbb{R} X$ une isotopie de surfaces compactes, alors cette fonction est la même pour tout $\left(X, \omega_{t}, c_{X}, B_{t}\right)$.

Remarquons qu'en particulier $\Gamma_{r}^{d, B}(J, \underline{x})$ ne dépend pas de la position relative de $\underline{x}$ par rapport à $B$, que $\Gamma_{r}^{d, B}=-\Gamma_{r}^{d, \mathbb{R} X \backslash B}$ et que le cas particulier où $B$ est vide est admissible et fournit un invariant que l'on a préalablement introduit dans [27]. Montrer l'invariance de $\Gamma_{r}^{d, \emptyset}(J, \underline{x})$ se trouve être une étape importante dans la démonstration de l'invariance de $\Gamma_{r}^{d, B}(J, \underline{x})$.

Théorème 2.2 ([28]) Sous les hypothèses du Théorème 2.1, si $B$ est un disque, $2 \chi_{r+1}^{d}=$ $\Gamma_{r}^{d, B}-\Gamma_{r}^{d, \emptyset}$. Si de plus, $\left(X, \omega, c_{X}\right)$ est symplectomorphe au plan projectif complexe, $\Gamma_{r}^{d, B}=$ $-\Gamma_{r}^{d, \emptyset}$, tandis que si elle est symplectomorphe à la quadrique hyperboloïde de dimension deux, $\Gamma_{r}^{d, B}=2 \chi_{r+1}^{d}-\Gamma_{r}^{d, \emptyset}$. 
Corollaire 6 ([28]) Sous les hypothèses du Théorème 2.2. $\chi_{r+1}^{d}=-\Gamma_{r}^{d, \emptyset}=\Gamma_{r}^{d, B}$ dans le cas du plan projectif complexe et $\Gamma_{r}^{d, B}=2 \chi_{r+1}^{d}, \Gamma_{r}^{d, \emptyset}=0$ dans le cas de la quadrique hyperboloïde de dimension deux.

\subsubsection{Sur les 3264 coniques tangentes à cinq coniques génériques}

Il est possible d'étendre les résultats du 2.1 .1 à davantage de conditions de tangence avec le bord de $B$, au moins dans le cas de coniques. J'ai illustré ce phénomène en m'intéressant au problème ancien du comptage du nombre de coniques tangentes à cinq coniques génériques données. Le nombre de solutions complexes est 3264 comme l'a démontré de Joncquières en 1859 mais le nombre de solutions réelles dépend du choix des cinq coniques génériques. Soient $B_{1}, \ldots, B_{5}$ cinq disques plongés dans $\mathbb{R} P^{2}$ de sorte que leurs bords soient transverses deux à deux, et $J \in \mathbb{R} \mathcal{J}_{\omega}$. Notons $\Gamma^{B}$ le nombre de coniques $J$-holomorphes réelles qui sont soit :

- irréductibles, tangentes à $B_{1}, \ldots, B_{5}$, et comptées positivement si elles sont tangentes intérieurement à $B_{i}$ pour un nombre pair de $i \in\{1, \ldots, 5\}$, négativement sinon.

- réductibles, tangentes à quatre des cinq disques $B_{1}, \ldots, B_{5}$, et chacune comptée en fonction de la parité du nombre de disques en lesquelles elle est tangente intérieurement et de la position de son unique point singulier par rapport au cinquième disque en lequel elle n'est pas tangente.

Ainsi, en notant respectivement $\mathcal{C}$ on $(J)$ et $\mathcal{C}_{\text {on }}$ red $(J)$ ces deux ensembles finis de coniques $J$-holomorphes, on obtient

$$
\Gamma^{B}(J)=\sum_{C \in \mathcal{C} \text { on }(J)}\langle C, B\rangle-\sum_{C \in \mathcal{C}_{\text {red }}(J)}\langle C, B\rangle \operatorname{mult}_{B}(C) \in \mathbb{Z},
$$

où lorsque $C \in \mathcal{C}$ on $(J)$, l'indice de contact $\langle C, B\rangle$ vaut $\prod_{i=1}^{5}\left\langle C, B_{i}\right\rangle$; tandis que lorsque $C \in \mathcal{C}_{\text {on }}$ red $(J)$ et $i_{1}, \ldots, i_{4} \in\{1, \ldots, 5\}$ sont les entiers tels que $C$ soit tangent aux bords de $B_{i_{1}}, \ldots, B_{i_{4}},\langle C, B\rangle=\Pi_{j=1}^{4}\left\langle C, B_{i_{j}}\right\rangle$ et $\operatorname{mult}_{B}(C)=+1$ si le point singulier de $C$ appartient à $B_{i_{5}}$ et -1 sinon.

Théorème 2.3 ([28]) 1) Cet entier $\Gamma^{B}(J)$ ne dépend pas du choix générique de la structure presque-complexe $J \in \mathbb{R} \mathcal{J}_{\omega}$ et est invariant par isotopie de $B=B_{1} \cup \cdots \cup B_{5}$.

2) Si $B_{1}, \ldots, B_{5}$ sont cinq disques disjoints, alors $\Gamma^{B}=272$. Il en est de même si $B_{1}, \ldots, B_{5}$ sont proches de cinq droites doubles génériques.

Un disque est dit proche d'une droite double d'équation $y^{2}=0$ s'il a une équation de la forme $\left\{y^{2} \leq \epsilon^{2} x^{2}-\delta\right\}$ pour $\epsilon$ et $\delta$ petits.

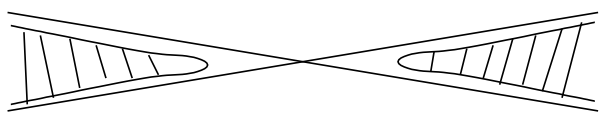


Corollaire 7 ([28]) Si $C_{1}, \ldots, C_{5}$ sont cinq coniques dont la classe d'isotopie est donnée par la deuxième partie du Théorème 2.3, alors le nombre de coniques réelles qui leur sont tangentes est minoré par 32 indépendamment du choix de $C_{1}, \ldots, C_{5}$ dans la classe d'isotopie.

\section{Démonstration :}

Le nombre de droites tangentes à deux coniques génériques vaut quatre, elles sont codées par les points d'intersection entre les deux coniques duales. Le nombre de coniques tangentes à quatre des cinq coniques $C_{1}, \ldots, C_{5}$ se trouve donc majoré par $240=5 * 3 * 4 * 4$, de sorte que le résultat découle de la définition de $\Gamma^{B}$ et de la deuxième partie du Théorème 2.3 .

Remarquons que le fait qu'il existe une configuration de cinq coniques réelles pour lesquelles les 3264 coniques tangentes à ces cinq coniques sont toutes réelles a été établi par F. Ronga, A. Tognoli et T. Vust [19]. Le théorème 2.1 montre la difficulté à définir des invariants relatifs avec conditions de tangence réelles. Dans le « monde tropical », la situation est parfois bien meilleure, voir [13]. Il en est de même avec des conditions de tangence complexes conjuguées, voir le $\$ 2.2$.

\subsection{Invariants relatifs imaginaires}

Soit $L$ une sphère, un tore ou un espace projectif réel de dimension $n=2$ ou 3 . Le fibré cotangent de $L$ est équipé de sa forme de Liouville $\lambda$ et de l'involution $c_{L}$ définie par $(q, p) \in T^{*} L \mapsto(q,-p) \in T^{*} L$. Cette dernière satisfait $c_{L}^{*} \lambda=-\lambda$ de sorte que $\left(T^{*} L, d \lambda, c_{L}\right)$ est une variété symplectique réelle. Soit $g$ une métrique à courbure constante sur $L, U^{*} L$ l'ensemble des couples $(q, p) \in T^{*} L$ tels que $g(p, p) \leq 1$ et $S^{*} L$ le bord de $U^{*} L$. La restriction de $\lambda$ à $S^{*} L$ est une forme de contact et l'on note $R_{\lambda}$ le champ de Reeb associé. Le flot engendré par $R_{\lambda}$ n'est autre que le flot géodésique. Notons $\mathcal{J}_{\lambda}$ l'espace des structures presque-complexes positives pour $d \lambda$ et asymptotiquement cylindriques sur une structure $C R$ de $S^{*} L$. Plus précisément, le champ radial de $T^{*} L$ identifie le complémentaire de la section nulle avec la symplectisation $\left(\mathbb{R} \times S^{*} L, d\left(e^{\rho} \lambda\right)\right)$ de $\left(S^{*} L, \lambda\right)$. On note $\mathcal{J}_{\lambda}$ l'espace des structures presque-complexes $J$ positives pour $d \lambda$, de classe $C^{l}, l \gg 1$, qui satisfont $J\left(\frac{\partial}{\partial \rho}\right)=R_{\lambda}$ et préservent le noyau de $\lambda$ pour $\rho \gg 1$ et qui enfin sont invariantes par translation par $\rho$ au-delà d'un certain rang $\rho_{0}$. Nous notons alors $\mathbb{R} \mathcal{J}_{\lambda} \subset \mathcal{J}_{\lambda}$ le sous-espace des structures presque-complexes pour lesquelles $c_{L}$ est $J$-antiholomorphe. Ces espaces $\mathcal{J}_{\lambda}$ et $\mathbb{R} \mathcal{J}_{\lambda}$ sont tous deux des variétés de Banach séparables non-vides et contractiles. Nous allons compter les courbes $J$-holomorphes rationnelles réelles pointées d'énergie de Hofer finie proprement immergées dans $T^{*} L$ en fonction d'un signe \pm 1 de façon à obtenir un invariant associé à $T^{*} L$. Rappelons que d'après le Théorème 1.2 de [11 et d'après [3], ces courbes rationnelles pointées convergent en leurs pointes vers des orbites de Reeb parcourues un nombre entier de fois, que l'on appelle multiplicité. La dimension de l'espace des modules de telles courbes dépend du nombre de pointes et des multiplicités associées. Afin d'obtenir un nombre fini de courbes, nous allons soumettre ces courbes à quelques 
contraintes, soit en les forçant à converger vers des orbites de Reeb prescrites, soit en les forçant à passer par des points de $L$ ou des paires de points complexes conjuguées de $T^{*} L \backslash L$.

Soit $e_{i}, i \geq 1$, la suite d'entiers partout nulle sauf au $i$-ème rang où elle vaut un. Soient $\alpha=\sum_{i \in \mathbb{N}^{*}} \alpha_{i} e_{i}$ et $\beta=\sum_{i \in \mathbb{N}^{*}} \beta_{i} e_{i}$ deux suites d'entiers positifs qui s'annulent à partir d'un certain rang. Ces deux suites codent respectivement le nombre de paires d'orbites de Reeb complexes conjuguées limites prescrites et non prescrites de nos courbes, avec leur multiplicités $i \in \mathbb{N}^{*}$. Le nombre de pointes de nos courbes vaut donc $2 v=2 \sum_{i \in \mathbb{N}^{*}}\left(\alpha_{i}+\beta_{i}\right)$ et nous choisissons un ensemble $\Gamma$ de $\sum_{i \in \mathbb{N}^{*}} \alpha_{i}$ géodésiques fermées disjointes de $L$ pour prescrire nos paires d'orbites de Reeb limites. À présent, afin de fixer nos contraintes ponctuelles, soient $r \in \mathbb{N}$ et $x_{1}, \ldots, x_{r}$ des points distincts de $L$. De même, soient $r_{L} \in \mathbb{N}$ et $\xi_{1}, \bar{\xi}_{1}, \ldots, \xi_{r_{L}}, \bar{\xi}_{r_{L}}$ des paires distinctes de points complexes conjugués de $T^{*} L \backslash L$, c'està-dire satisfaisant $c_{L}\left(\xi_{i}\right)=\bar{\xi}_{i}$. Nous supposons que

$$
(n-1) r+2(n-1) r_{L}+2(n-1) \# \Gamma=2 v+\epsilon(n-1) \sum_{i \in \mathbb{N}^{*}} i\left(\alpha_{i}+\beta_{i}\right)+n-3,
$$

où $\epsilon=2$ si $L$ est homéomorphe à une sphère et $\epsilon=1$ si $L$ est homéomorphe à un espace projectif réel, tandis que nous supposons

$$
(n-1) r+2(n-1) r_{L}=2 v+n-3 \text { et } \alpha=0
$$

si $L$ est homéomorphe à un tore.

Alors, lorsque la structure presque-complexe $J \in \mathbb{R} \mathcal{J}_{\lambda}$ est générique, il n'y a qu'un nombre fini de courbes $J$-holomorphes rationnelles réelles d'énergie de Hofer finie, proprement immergées dans $T^{*} L$ et ayant $2 v$ pointes qui passent par $\underline{x}$, par chaque paire $\left\{\xi_{i}, \bar{\xi}_{i}\right\}$ et qui convergent vers les orbites de Reeb relevant les éléments de $\Gamma$ ainsi que vers $\beta_{j}$ autres paires d'orbites, $j \in \mathbb{N}^{*}$, chacune avec multiplicité $j$ ou de classe d'homologie donnée si $L$ est un tore. En effet, si $L$ est un tore, il y a une infinité de géodésiques fermées primitives non homologues et la dimension (2) ne dépend pas du choix des classes d'homologies de sorte qu'il y a une infinité d'espaces de modules ayant la même dimension. Pour garantir la finitude, nous imposons les classes d'homologies des orbites de Reeb limites. Notons $\mathcal{R}(\alpha, \beta, \Gamma, \underline{x}, \underline{\xi}, J)$ cet ensemble fini de courbes, la généricité de $J$ garantit qu'elles sont toutes immergées. Si $L$ est de dimension deux, on pose

$$
F_{\left(r, r_{L}\right)}(\alpha, \beta, \Gamma, \underline{x}, \underline{\xi}, J)=\sum_{C \in \mathcal{R}(\alpha, \beta, \Gamma, \underline{x}, \underline{\xi}, J)}(-1)^{m(C)} \in \mathbb{Z} .
$$

Si $L$ est de dimension trois, on l'équipe d'une structure spin. Ceci permet d'associer un état spinoriel $\operatorname{sp}(C)$ à chaque courbe $C \in \mathcal{R}(\alpha, \beta, \Gamma, \underline{x}, \underline{\xi}, J)$ comme expliqué au 33.1 et on pose

$$
F_{\left(r, r_{L}\right)}(\alpha, \beta, \Gamma, \underline{x}, \underline{\xi}, J)=\sum_{C \in \mathcal{R}(\alpha, \beta, \Gamma, \underline{x}, \underline{\xi}, J)} \operatorname{sp}(C) \in \mathbb{Z} .
$$


Théorème 2.4 ([30]) Soit $L$ une sphère, un tore ou un espace projectif réel de dimension $n=2$ ou 3 muni d'une métrique à courbure constante. Soient $\alpha, \beta$ deux suites d'entiers positifs qui s'annulent à partir d'un certain rang. On choisit comme ci-dessus un ensemble $\Gamma$ de géodésiques fermées et des ensembles $\underline{x}, \underline{\xi}$ de $r$ et $r_{L}$ points dans $L$ et $T^{*} L \backslash L$ respectivement de sorte que ces nombres satisfassent (2) dans le cas du tore et (1) sinon. Lorsque $n=3$, on suppose $r \neq 0$ et lorsque de plus $L \in\left\{S^{3}, \mathbb{R} P^{3}\right\}$, on suppose que $J$ est invariante par le flot de Reeb pour $\rho \gg 1$. Alors, l'entier $F_{\left(r, r_{L}\right)}(\alpha, \beta, \Gamma, \underline{x}, \xi, J)$ défini ci-dessus ne dépend ni du choix des contraintes $\Gamma, \underline{x}, \underline{\xi}$, ni du choix générique de la structure presque-complexe $J \in \mathbb{R} \mathcal{J}_{\lambda}$.

L'entier $F_{\left(r, r_{L}\right)}(\alpha, \beta, \Gamma, \underline{x}, \underline{\xi}, J)$ étant indépendant de $\Gamma, \underline{x}, \underline{\xi}, J$ d'après le Théorème 2.4 . nous le noterons $F_{\left(r, r_{L}\right)}(\alpha, \beta)$. Afin d'alléger encore cette notation, nous noterons cet entier $F(\alpha, \beta)$ lorsque $r_{L}=0$, puisque la valeur de $r$ est alors définie sans ambiguïté par les calculs de dimensions (1) et (2). Les Lemmes 2.5, 2.6 et 2.7 fournissent quelques calculs que l'on a pu mener. Les résultats du $\$ 1.4$ reposent sur ces calculs.

Lemme 2.5 ([30]) Si $L$ est homéomorphe à une sphère de dimension deux et $r_{L}=0$, on a $F\left(e_{1}, 0\right)=F\left(0, e_{1}\right)=1, F\left(e_{2}, 0\right)=2, F\left(0, e_{2}\right)=8, F\left(2 e_{1}, 0\right)=2, F\left(e_{1}, e_{1}\right)=4$ et $F\left(0,2 e_{1}\right)=6$.

Lemme 2.6 ([30]) Si $L$ est homéomorphe à un plan projectif réel et $r_{L}=0$, on a $F\left(e_{1}, 0\right)=F\left(0, e_{1}\right)=F\left(e_{2}, 0\right)=F\left(2 e_{1}, 0\right)=F\left(e_{1}, e_{1}\right)=F\left(0,2 e_{1}\right)=1$ et $F\left(0, e_{2}\right)=4$.

Lemme 2.7 ([30]) Si $L$ est homéomorphe à un plan projectif réel et $r_{L}=0$, on a $F\left(e_{3}, 0\right)=2, F\left(0, e_{3}\right)=12, F\left(e_{1}+e_{2}, 0\right)=2, F\left(e_{1}, e_{2}\right)=8, F\left(e_{2}, e_{1}\right)=4, F\left(0, e_{1}+e_{2}\right)=$ $24, F\left(3 e_{1}, 0\right)=2, F\left(2 e_{1}, e_{1}\right)=4, F\left(e_{1}, 2 e_{1}\right)=6$ et $F\left(0,3 e_{1}\right)=8$.

Toutefois, la valeur de l'invariant $F$ qui ressort du Théorème 2.4 n'est pas connue en général. Il serait intéressant de développer des méthodes permettant son calcul.

\section{Invariants en dimension six}

Nous exposons dans ce paragraphe les résultats analogues à ceux présentés au \$1 que l'on a pu établir en dimension six.

\subsection{Définition des invariants dans les variétés algébriques réelles convexes}

Rappelons qu'une variété projective lisse est dite convexe lorsque le groupe $H^{1}\left(\mathbb{C} P^{1} ; u^{*} T X\right)$ s'annule pour tout morphisme $u: \mathbb{C} P^{1} \rightarrow X$. Les principaux exemples que je connaisse sont les espaces homogènes projectifs, citons les produits d'espaces projectifs, la quadrique de $\mathbb{C} P^{4}$ ou encore la variété des drapeaux de $\mathbb{C}^{3}$. Il est à nouveau possible de définir un invariant en comptant algébriquement le nombre de courbes rationnelles réelles qui 
réalisent une classe d'homologie $d$ donnée et passent par une configuration réelle de points $\underline{x}$ de cardinal $\frac{1}{2} c_{1}(X) d$, où $c_{1}(X)$ désigne la première classe de Chern de la variété et $c_{1}(X) d$ est supposé pair. Toutefois, le signe \pm 1 en fonction duquel il convient de compter les courbes rationnelles réelles est plus délicat à définir. Le lieu réel $\mathbb{R} X=\operatorname{fixe}\left(c_{X}\right)$ de $X$ est une variété lisse de dimension réelle trois que l'on suppose orientable pour simplifier. Munissons-la d'une orientation ainsi que d'une métrique riemannienne auxiliaire. Son $\mathrm{SO}_{3}(\mathbb{R})$-fibré principal des repères orthonormés directs s'étend alors en un $\mathrm{Spin}_{3}$-fibré principal. En effet, l'obstruction à l'existence d'une telle extension est donnée en général par la classe caractéristique $w_{2}(\mathbb{R} X)$ et cette obstruction s'annule en dimension trois comme il découle de la formule de $\mathrm{Wu}$. Lorsque la configuration réelle de points est suffisamment générique et possède au moins un point réel, d'une part les courbes rationnelles réelles qui passent par $\underline{x}$ et réalisent $d$ sont toutes immergées (même lisses en général) et de partie réelle non vide, et d'autre part elle sont équilibrées. Ce dernier point signifie que le fibré normal de ces courbes se décompose sur $\mathbb{C}$ comme la somme directe de deux fibrés en droite isomorphes $L$ et $M$, fibrés qui de plus peuvent être choisis réels. Notons $\mathcal{R}_{d}(\underline{x})$ cet ensemble fini de courbes rationnelles réelles. Chaque lieu réel de ces courbes fournit donc un nœud immergé dans la variété de dimension trois $\mathbb{R} X$, et ce nœud est de plus canoniquement équipé d'un repère mobile ou plutôt d'axes mobiles donnés par la tangente au nœud et les lieux réels des fibrés en droites $L$ et $M$ (en fait, seule la classe d'homotopie de ces axes mobiles est canoniquement définie, puisque la décomposition du fibré normal en somme $L \oplus M$ n'est pas uniquement définie, mais c'est amplement suffisant pour nos besoins). Lorsque les lieux réels de $L$ et $M$ sont orientables, c'est-à-dire lorsque ces fibrés sont de degré pair, ces axes mobiles peuvent être enrichis de repères orthonormés. Ainsi, les nœuds définis par les courbes rationnelles réelles sont tous équipés de repères orthonormés mobiles qui fournissent des lacets dans le $\mathrm{SO}_{3}(\mathbb{R})$-fibré principal des repères orthonormés, lacets qui relèvent les nœuds de $\mathbb{R} X$. Vient alors l'alternative suivante pour chaque lacet : soit ce lacet du $\mathrm{SO}_{3}(\mathbb{R})$-fibré principal des repères se relève en un lacet du $\mathrm{Spin}_{3}$-fibré principal donné par la structure Spin $\mathfrak{s}$, soit non. Ceci permet de définir l'état spinoriel $\operatorname{sp}(C)$ de chaque courbe rationnelle réelle $C$ comme valant +1 dans le premier cas, et -1 dans le second. Lorsque les lieux réels de $L$ et $M$ ne sont pas orientables, on modifie ces axes mobiles à l'aide d'un demi-tour à droite donné par l'orientation de $\mathbb{R} X$, ce qui permet de se ramener au cas précédent et de définir l'état spinoriel également dans ce cas. L'entier $\chi_{r}^{d, \mathfrak{s}}(\underline{x})$ n'est alors autre que le nombre de courbes rationnelles réelles qui réalisent la classe d'homologie $d$ et passent par $\underline{x}$, ces courbes étant comptées en fonction de leur état spinoriel, de sorte que

$$
\chi_{r}^{d, \mathfrak{s}}(\underline{x})=\sum_{C \in \mathcal{R}_{d}(\underline{x})} s p(C) \in \mathbb{Z} .
$$

On a noté $r=\left(r_{1}, \ldots, r_{N}\right)$ le $N$-uplet associé à $\underline{x}$; c'est-à-dire que $N$ désigne le nombre de composantes connexes de $\mathbb{R} X$ et en notant $(\mathbb{R} X)_{1}, \ldots,(\mathbb{R} X)_{N}$ ces composantes, $r_{i}=$ $\#\left(\underline{x} \cap(\mathbb{R} X)_{i}\right)$.

Théorème $3.1([26])$ Soient $\left(X, c_{X}\right)$ une variété algébrique réelle convexe lisse de dimension trois et $\mathfrak{s}$ une structure $S_{p i n}$ sur son lieu réel $\mathbb{R} X$ supposé orientable. Soit 
$d \in H_{2}(X ; \mathbb{Z})$ telle que $c_{1}(X) d$ soit pair et différent de quatre et soit $k_{d}=\frac{1}{2} c_{1}(X) d \in \mathbb{N}^{*}$. Soient $\underline{x}=\left(x_{1}, \ldots, x_{k_{d}}\right)$ une configuration réelle de $k_{d}$ points distinct dont au moins un réel et $r$ le $N$-uplet associé. L'entier $\chi_{\underline{r}}^{d, \mathfrak{s}}(\underline{x})$ ne dépend alors pas du choix générique de $\underline{x}$.

Ce résultat est valable aussi pour les variétés dont le lieu réel n'est pas orientable, moyennant le choix d'une structure $\operatorname{Pin}_{3}^{-}$sur le lieu réel ; l'invariant s'annule alors lorsque $k_{d}$ est pair, voir [26].

Le Théorème 3.1 permet sans ambiguité de noter cet entier $\chi_{r}^{d, \mathfrak{s}}$. Lorsque $\sum_{i=1}^{N} r_{i}$ n'a pas la même parité que $k_{d}=\frac{1}{2} c_{1}(X) d$, on pose $\chi_{r}^{d, \mathfrak{s}}=0$. On note alors $\chi^{d, \mathfrak{s}}[T]$ la fonction génératrice $\sum_{|r|=0}^{k_{d}} \chi_{r}^{d, \mathfrak{s}} T^{r} \in \mathbb{Z}\left[T_{1}, \ldots, T_{N}\right]$. Cette fonction est de même parité que $\frac{1}{2} c_{1}(X) d$ et tous ses monômes ne dépendent en fait que d'une indéterminée.

Ainsi, la fonction $\chi^{\mathfrak{s}}: d \in H_{2}(X ; \mathbb{Z}) \mapsto \chi^{d, \mathfrak{s}}(T) \in \mathbb{Z}[T]$ est invariante par isomorphisme de la variété algébrique réelle convexe lisse de dimension trois $\left(X, c_{X}\right)$. On en déduit à nouveau les bornes inférieures suivantes en géométrie énumérative réelle.

Corollaire 8 ([26]) Sous les hypothèses du Théorème 3.1, notons $R_{d}(\underline{x})$ le nombre de courbe rationnelles réelles connexes homologues à d qui passent par $\underline{x}$ et $N_{d}$ l'invariant de Gromov-Witten de genre zéro associé. Alors, $\left|\chi_{r}^{d, \mathfrak{s}}\right| \leq R_{d}(\underline{x}) \leq N_{d}$.

Finissons ce paragraphe par une interprétation topologique de nos résultats. Les singularités de l'espace $\mathbb{R}_{\tau} \overline{\mathcal{M}}_{k_{d}}^{d}(X)$ sont de codimension au moins deux, de sorte que cet espace possède une première classe de Stiefel-Whitney. Étant donné $D \in H_{3 k_{d}-1}\left(\mathbb{R}_{\tau} \overline{\mathcal{M}}_{k_{d}}^{d}(X) ; \mathbb{Z} / 2 \mathbb{Z}\right)$, on note $D^{\vee}$ son image sous le morphisme $H_{3 k_{d}-1}\left(\mathbb{R}_{\tau} \overline{\mathcal{M}}_{k_{d}}^{d}(X) ; \mathbb{Z} / 2 \mathbb{Z}\right) \rightarrow H^{1}\left(\mathbb{R}_{\tau} \overline{\mathcal{M}}_{k_{d}}^{d}(X) ; \mathbb{Z} / 2 \mathbb{Z}\right)$.

Proposition $3.2([24])$ La première classe de Stiefel-Whitney de toute composante $\mathbb{R} \mathcal{M}^{*}$ de $\mathbb{R}_{\tau} \mathcal{M}_{k_{d}}^{d}(X)$ qui contient une courbe équilibrée s'écrit

$$
w_{1}\left(\mathbb{R} \mathcal{M}^{*}\right)=\left(\mathbb{R}_{\tau} e v^{d}\right)^{*} w_{1}\left(\mathbb{R}_{\tau} X^{k_{d}}\right)+\sum_{D \subset R e d^{\prime}} \epsilon(D) D^{\vee} \in H^{1}\left(\mathbb{R} \mathcal{M}^{*} ; \mathbb{Z} / 2 \mathbb{Z}\right)
$$

où $\epsilon(D) \in\{0,1\}$ et lorsque $\epsilon(D)=1$, la composante irréductible $D$ de Red se trouve contractée par l'application d'évaluation $\mathbb{R}_{\tau} e v^{d}$.

On a noté ici Red' la réunion du diviseur des courbes réductibles Red et de l'éventuel diviseur des courbes non-équilibrées $(u, C, \underline{z})$ telles que $\operatorname{dim} H^{1}\left(C ; N_{u} \otimes \mathcal{O}_{C}(-\underline{z})\right) \geq 2$, si un tel diviseur existe. On note $\operatorname{Red}_{1}$ la réunion des composantes irréductibles $D$ de $\operatorname{Red}^{\prime}$ pour lesquelles $\epsilon(D)=1$. En dimension deux, cet ensemble a été déterminé dans [18]. Équipons $\mathbb{R}_{\tau} X^{k_{d}}$ d'un système de coefficients tordus entiers $\mathcal{Z}$ et notons $\left[\mathbb{R}_{\tau} X^{k_{d}}\right] \in H_{3 k_{d}}\left(\mathbb{R}_{\tau} X^{k_{d}} ; \mathcal{Z}\right)$ sa classe fondamentale. Notons $\mathcal{Z}^{*}$ le système de coefficients locaux induit sur $\mathbb{R} \mathcal{M}^{*}$, tiré en arrière de $\mathcal{Z}$ par $\mathbb{R}_{\tau} e v^{d}$.

Proposition 3.3 ([24]) Sous les hypothèses de la Proposition 3.2, il existe une unique classe fondamentale $\left[\mathbb{R} \mathcal{M}^{*}\right] \in H_{3 k_{d}}\left(\mathbb{R} \mathcal{M}^{*}, \operatorname{Red}_{1} ; \mathcal{Z}^{*}\right)$ telle qu'en toute courbe équilibrée 
$(u, C, \underline{z}) \in \mathbb{R} \mathcal{M}^{*}$, le morphisme

$$
\left(\mathbb{R}_{\tau} e v^{d}\right)_{*}: H_{3 k_{d}}\left(\mathbb{R}^{*}, \mathbb{R}^{*} \backslash\{(u, C, \underline{z})\} ; \mathcal{Z}^{*}\right) \rightarrow H_{3 k_{d}}\left(\mathbb{R}_{\tau} X^{k_{d}}, \mathbb{R}_{\tau} X^{k_{d}} \backslash\{u(\underline{z})\} ; \mathcal{Z}\right)
$$

envoie $\left[\mathbb{R} \mathcal{M}^{*}\right] \operatorname{sur} \operatorname{sp}(u, C, \underline{z})\left[\mathbb{R}_{\tau} X^{k_{d}}\right]$.

Comme $\mathbb{R}_{\tau} e v^{d}\left(\operatorname{Red}_{1}\right)$ est de codimension deux, le groupe $H_{3 k_{d}}\left(\mathbb{R}_{\tau} X^{k_{d}}, \mathbb{R}_{\tau} e v^{d}\left(\operatorname{Red}_{1}\right) ; \mathcal{Z}\right)$ est cyclique, engendré par $\left[\mathbb{R}_{\tau} X^{k_{d}}\right]$. L'entier $\chi_{r}^{d, 5}$ n'est autre que celui défini par la relation $\left(\mathbb{R}_{\tau} e v^{d}\right)_{*}\left[\mathbb{R}_{\tau} \overline{\mathcal{M}}_{k_{d}}^{d}(X)\right]=\chi_{r}^{d, 5}\left[\mathbb{R}_{\tau} X^{k_{d}}\right]$, où la classe fondamentale $\left[\mathbb{R}_{\tau} \overline{\mathcal{M}}_{k_{d}}^{d}(X)\right]$ est donnée par la Proposition 3.3 .

\subsection{Extension aux variétés symplectiques réelles fortement semi- positives}

L'extension des résultats du $\$ 3.1$ aux variétés symplectiques n'est pas immédiate, en partie parce-que le thérorème de Grothendieck [10] selon lequel les fibrés holomorphes sur la sphère de Riemann sont entièrement décomposables n'est plus valable pour les fibrés normaux des courbes pseudo-holomorphes. Ces derniers sont des fibrés vectoriels complexes munis d'un opérateur de Cauchy-Riemann qui n'est que $\mathbb{R}$-linéaire et non $\mathbb{C}$-linéaire comme dans le cas de fibrés holomophes. Ces premiers sont des perturbations d'ordre zéro de ces derniers par des opérateurs $\mathbb{C}$-antilinéaires et sont parfois appelés « opérateurs de CauchyRiemann généralisés ». J'ai étendu dans [24] la notion d'état spinoriel pour un opérateur de Cauchy-Riemann généralisé surjectif.

La stratégie est la suivante. L'espace des opérateurs de Cauchy-Riemann généralisés réels sur un fibré vectoriel complexe réel donné est un espace de Banach affine, il contient les opérateurs de Cauchy-Riemann $\mathbb{C}$-linéaires comme sous-espace de Banach. Or chaque opérateur de Cauchy-Riemann surjectif définit une structure de fibré vectoriel holomorphe équilibré et possède donc un état spinoriel d'après les résultats du \$3.1. Étant donné un opérateur de Cauchy-Riemann généralisé surjectif, on le relie à opérateur de CauchyRiemann surjectif par un chemin générique et on définit son état spinoriel comme celui de l'opérateur de Cauchy-Riemann si le chemin traverse un nombre pair de fois le mur des opérateurs non-surjectifs et son opposé sinon.

Soit alors $\left(X, \omega, c_{X}\right)$ une variété symplectique réelle fortement semi-positive de dimension six, c'est-à-dire pour laquelle toute classe sphérique $d \in H_{2}(X ; \mathbb{Z})$ positive contre $\omega$ satisfait l'implication $c_{1}(X) d \geq 2-n \Longrightarrow c_{1}(X) d \geq 1$. Les variétés symplectiques réelle positives, par exemple de Fano, satisfont cette condition. On suppose à nouveau pour simplifier le lieu réel de cette variété orientable et on l'équipe d'une structure spin $\mathfrak{s}$. Soit, comme au $\$ 3.1, d \in H_{2}(X ; \mathbb{Z})$ telle que $\left(c_{X}\right)_{*} d=-d, c_{1}(X) d$ soit pair et strictement plus grand que deux. Soient $k_{d}=\frac{1}{2} c_{1}(X) d$ et $\underline{x}=\left(x_{1}, \ldots, x_{k_{d}}\right) \in X^{k_{d}}$ une configuration réelle de $k_{d}$ points distincts, dont au moins un réel. Lorsque $J \in \mathbb{R} \mathcal{J}_{\omega}$ est suffisamment générique, il n'y a qu'un nombre fini de courbes $J$-holomorphes rationnelles réelles connexes homologues à $d$ et contenant $\underline{x}$. Ces courbes sont toutes irréductibles, lisses et de partie réelle non-vide. On note $\mathcal{R}_{d}(\underline{x}, J)$ cet ensemble fini de courbes. Le fibré normal de chacune de ces courbes 
$C \in \mathcal{R}_{d}(\underline{x}, J)$ est équipé d'un opérateur de Cauchy-Riemann généralisé surjectif $D_{C}$ qui possède donc un état spinoriel $s p(C)$ d'après ce qui précède. On pose

$$
\chi_{r}^{d, \mathfrak{s}}(\underline{x}, J)=\sum_{C \in \mathcal{R}_{d}(\underline{x}, J)} s p(C) \in \mathbb{Z} .
$$

Théorème $3.4([24])$ Soit $\left(X, \omega, c_{X}\right)$ une variété symplectique réelle fortement semi-positive de dimension six, de lieu réel orientable muni d'une structure spin $\mathfrak{s}$. Soit $d \in H_{2}(X ; \mathbb{Z})$ telle que $\left(c_{X}\right)_{*} d=-d, c_{1}(X) d$ est pair et strictement plus grand que deux. Soient $k_{d}=$ $\frac{1}{2} c_{1}(X) d$ et $\underline{x}$ une configuration réelle de $k_{d}$ points distincts, dont au moins un réel. et $r=\left(r_{1}, \ldots, r_{N}\right)$ le $N$-uplet associé. Alors, l'entier $\chi_{r}^{d, \mathfrak{s}}(\underline{x}, J)$ ne dépend ni du choix de $\underline{x}$, ni du choix générique de $J \in \mathbb{R} \mathcal{J}_{\omega}$.

Remarquons que ce résultat permet de noter sans ambiguité l'invariant $\chi_{r}^{d, \mathfrak{s}}$, c'est un invariant par déformation fortement semipositive de $\left(X, \omega, c_{X}\right)$. On en déduit les bornes inférieures suivantes.

Corollaire 9 ([24]) Sous les hypothèses du Théorème 3.4. $\left|\chi_{r}^{d, \mathfrak{s}}\right| \leq \# \mathcal{R}_{d}(\underline{x}, J)$, pour tout choix de configuration réelle $\underline{x} \in X^{k_{d}}$ telle que $\underline{x} \cap \mathbb{R} X=r$, et tout choix générique de $J \in \mathbb{R} \mathcal{J}_{\omega}$.

Les invariants qui ressortent des Théorèmes 1.1 et 3.1 ont été interprétés par C.-H. Cho [5] et J. Solomon [22]. Leur approche consiste à d'abord définir la classe fondamentale $\left[\mathbb{R}_{\tau} \overline{\mathcal{M}}_{k_{d}}^{d}(X)\right]$ donnée par la Proposition 3.3 en utilisant les travaux de K. Fukaya, Y.-G. Oh, H. Ohta et K. Ono [6], [7], puis à en déduire l'existence des invariants grâce à la relation entre classes fondamentales donnée à la suite de cette proposition. J. Solomon a étendu ces invariants aux courbes de genre strictement positifs mais de structure conforme fixée et aux variétés symplectiques de dimension six, notamment de Calabi-Yau. Dans le cas des quintiques de $\mathbb{C} P^{4}$, l'invariant a été calculé par R. Pandharipande, J. Solomon et J. Walcher [17].

\subsection{Optimalité, congruences et calculs dans le cas de l'ellipsoïde de dimension trois}

Théorème 3.5 ([30]) Soient $\left(X, c_{X}\right)$ la quadrique ellipsoïde de dimension trois et $d \in$ $H_{2}(X ; \mathbb{Z})$ satisfaisant $c_{1}(X) d=2 \bmod (4)$. L'invariant $\chi_{1}^{d}$ est alors négatif et les bornes inférieures apparues dans le Corollaire 8 sont optimales, atteintes lorsque les conditions d'incidence non réelles sont choisies suffisamment proches d'une section hyperplane réelle disjointe du lieu réel $\mathbb{R} X$.

Remarque 4 La condition $c_{1}(X) d=2 \bmod (4)$ garantit la parité de l'entier $k_{d}$ de sorte que l'on peut effectivement choisir un point réel. Lorsque $c_{1}(X) d=0 \bmod (4)$, et $r=0$, 
l'invariant $\chi_{r}^{d}$ n'est pas défini. Toutefois, on a montré dans ce cas là qu'il existe une structure presque-complexe générique $J \in \mathbb{R} \mathcal{J}_{\omega}$ et $k_{d}$ points complexes conjugués pour lesquels aucune courbe $J$-holomorphe rationnelle réelle homologue à $d$ contient ces $k_{d}$ points, voir le Théorème 4.2.

Théorème 3.6 ([30]) Soient $\left(X, c_{X}\right)$ la quadrique ellipsoïde de dimension trois et $d$ un multiple positif, disons $\delta>0$, d'une section hyperplane réelle. Lorsque $6 r+1 \leq 3 \delta$, la puissance $2^{\frac{3}{4}(\delta-2 r)}$ divise $\chi_{r}^{d}$.

Corollaire 10 ([30]) Soit $\left(X, \omega, c_{X}\right)$ une variété symplectomorphe à la quadrique ellipsoïde de dimension trois. Alors, $\chi_{1}^{2}=-1, \chi_{1}^{6}=0$ et $\chi_{1}^{10}=-896$.

Dans le cas de l'espace projectif de dimension trois, une formule calculant $\chi_{2 d}^{d}\left(\mathbb{C} P^{3}\right)$ pour tout degré $d$ est annoncée par E. Brugallé et G. Mikhalkin dans [4]. En particulier, $\chi_{10}^{5}=45$ et $\chi_{14}^{7}=-14589$ alors qu'en degré pair, l'invariant s'annule pour des raisons de symétrie.

\section{Sur la présence et l'absence de membranes $J$-holomorphes}

\subsection{Absence de membranes $J$-holomorphes}

Soit $C$ une membrane $J$-holomorphe à bord dans une sous-variété lagrangienne $L$ d'une variété symplectique fermée $(X, \omega)$. Notons $\chi$ la caractéristique d'Euler de cette membrane, $d \in H_{2}(X, L ; \mathbb{Z})$ sa classe d'homologie relative et $\mu_{T X} \in H^{2}(X, L ; \mathbb{Z})$ la classe de Maslov de la paire $(X, L)$. La dimension attendue de l'espace des déformations de $C$ s'écrit $\left\langle\mu_{T X}, d\right\rangle+$ $(n-3) \chi$. Cette dimension chute lorsque l'on impose à $C$ des contraintes supplémentaires. Si l'on impose par exemple à cette membrane de rencontrer $p$ cycles de codimensions $2+$ $q_{1}, \ldots, 2+q_{p}$, cette dimension attendue chute de la somme $q=q_{1}+\cdots+q_{p}$. Deux problèmes généraux sous-tendent nos résultats. Il s'agit d'une part de compter les membranes $J$ holomorphes homologues à $d$ soumises à de telles conditions d'incidence de sorte que ce comptage ne dépende pas de $J$ et ne dépende des conditions d'incidence qu'à homologie près. Il s'agit d'autre part de minimiser ce nombre de membranes. Si nous ne pouvons répondre au premier problème dans ce degré de généralité, il nous est par contre parfois possible de répondre au second sans même supposer l'égalité $q=\left\langle\mu_{T X}, d\right\rangle+(n-3) \chi$, lorsque le minimum en question est nul. Le présent paragraphe est consacré aux résultats que l'on a pu obtenir dans cette direction. Ici encore le minimum est atteint en allongeant le cou d'une structure presque complexe générale.

\subsubsection{En dimension supérieure}

Théorème 4.1 ([30], [31]) Soit L une sphère lagrangienne dans une variété symplectique fermée $(X, \omega)$ satisfaisant $c_{1}(X)=\lambda \omega, \lambda \leq 0$ et soit $E>0$. Supposons la dimension de $X$ supérieure à cinq. Pour toute structure presque-complexe $J$ générale ayant un cou 
suffisamment long au voisinage de L, cette variété ne possède ni membrane J-holomorphe reposant sur $L$ ni courbe $J$-holomorphe rencontrant $L$ qui soit d'énergie inférieure à $E$. Ce résultat reste valable en dimension quatre pour les courbes ou membranes de genre nul.

Rappelons que l'énergie d'une courbe $C$ est par définition l'intégrale de la forme $\omega$ sur cette courbe. Les variétés projectives à fibré canonique nul ou ample, par exemple les intersections complètes de multidegrés $\left(d_{1}, \ldots, d_{k}\right)$ de l'espace projectif de dimension $N$ dès lors que $\sum_{i=1}^{k} d_{i} \geq N+1$, satisfont les hypothèses du Théorème 4.1. Remarquons qu'une modification de ce dernier s'applique également aux variétés dont le fibré canonique est le produit d'un fibré ample et d'un fibré porté par un diviseur effectif disjoint de $L$. Le Théorème 4.1 permet de définir la cohomologie de Floer de sphères lagrangiennes dans les variétés symplectiques dont la première classe de Chern s'annule, voir [31] et [6], [7] pour une théorie de l'obstruction à définir en général une telle homologie.

Théorème $4.2([30])$ Soit L une sphère lagrangienne dans une variété symplectique fermée semipositive $(X, \omega)$ de dimension $2 n \geq 6$ et soit $d \in H_{2}(X, L ; \mathbb{Z})$. Écrivons $\left\langle\mu_{T X}, d\right\rangle+(n-$ $3) \chi=q+r$ avec $q \in \mathbb{Z}, 0 \leq r<2+(n-3) \chi$ et $\chi \leq 2$. Lorsque $q \geq 0$, choisissons $p$ cycles de $X \backslash L$ de codimensions $2+q_{1}, \ldots, 2+q_{p}$ de sorte que $q=q_{1}+\cdots+q_{p}$. Dès que la structure presque complexe générale $J$ possède un cou suffisamment long au voisinage de L, cette variété ne contient aucune membrane J-holomorphe homologue à d, de caractéristique d'Euler $\chi$ qui rencontre ces p cycles et repose sur L. Ce résultat reste valable pour des membranes de genre nul lorsque $n=2$.

\section{Exemple : la quadrique ellipsoïde.}

Soit $X$ la quadrique ellipsoïde de dimension complexe $n \geq 3$ et $H$ une section hyperplane disjointe de $L$. Le groupe $H_{2}(X, L ; \mathbb{Z})$ est monogène, engendré par la classe $d_{0}$ satisfaisant $\left\langle H, d_{0}\right\rangle=+1$. La première classe de Chern de $X$ vaut $n H$, d'où l'on déduit le calcul $\left\langle\mu_{T X}, l d_{0}\right\rangle=2 \ln$ quel que soit l'entier $l$. Écrivons $l=(n-1) a+b$, le Théorème 4.2 s'applique par exemple lorsque $n+1 \leq 2 b<2 n$, les membranes sont des disques et lorsque toutes les conditions d'incidence sont ponctuelles. Rappelons que le Théorème 3.5 traite du cas $r=n-1$ et montre ainsi en un sens l'optimalité des hypothèses faites dans ce Théorème 4.2 .

\subsubsection{En dimension quatre}

Nous noterons $\mathcal{M}_{g, b}$ l'espace des modules des structures complexes de la surface compacte connexe orientée de genre $g$ ayant $b$ composantes de bord.

Proposition $4.3([30])$ Soit $L$ une sphère lagrangienne dans une variété symplectique fermée de dimension quatre $(X, \omega)$. On suppose que cette dernière ne possède pas de sphère symplectique $S$ satisfaisant $\left\langle c_{1}(X),[S]\right\rangle>0$. Soit $(d, g, b) \in H_{2}(X, L ; \mathbb{Z}) \times \mathbb{N} \times \mathbb{N}^{*}$ et $K$ un compact de $\mathcal{M}_{g, b}$. Alors, pour toute structure presque-complexe générale ayant un cou suffisamment long au voisinage de L, la variété ne possède pas de membrane J-holomorphe homologue à d à bord dans $L$ et conforme à un élément de $K$. 
Proposition 4.4 ([30]) Soit L une surface lagrangienne orientable hyperbolique dans une variété symplectique fermée de dimension quatre $(X, \omega)$ et soit $d \in H_{2}(X, L ; \mathbb{Z})$. On note $N_{d}^{g}(\underline{x}, J)$ le nombre de courbes $J$-holomorphes homologues à d à bords dans $L$, de topologie et de structure conforme données et qui passent par une configuration $\underline{x}$ de points distincts de $(X, \omega)$ de cardinal adéquat, pour $J \in \mathcal{J}_{\omega}$ générique. Ce nombre $N_{d}^{g}(\underline{x}, J)$ s'annule pour toute structure presque-complexe générale ayant un cou suffisamment long au voisinage de $L$.

Proposition $4.5([30])$ Soit $\left(X, \omega, c_{X}\right)$ une variété symplectique réelle fermée de dimension quatre dont le lieu réel possède un tore lagrangien ou bien une surface hyperbolique lagrangienne $L$, orientable ou non. On suppose que $\left(X, \omega, c_{X}\right)$ ne possède pas de sphère symplectique réelle $S$ satisfaisant $\left\langle c_{1}(X),[S]\right\rangle>1$ si $L$ est orientable et $\left\langle c_{1}(X),[S]\right\rangle>0$ sinon. Soit $(d, g, b) \in H_{2}(X, L ; \mathbb{Z}) \times \mathbb{N} \times \mathbb{N}^{*}$ et $K$ un compact de $\mathcal{M}_{g, b}$. Alors, pour toute structure presque-complexe générale ayant un cou suffisamment long au voisinage de $L$, la variété ne possède pas de membrane J-holomorphe homologue à d à bord dans $L$ et conforme à un élément de $K$.

\subsection{Présence de membranes $J$-holomorphes}

Les résultats présentés aux $\S \S 1.1$ et 3.1 permettent de garantir l'existence de disques $J$ holomorphes reposant sur une sous-variété lagrangienne d'une variété symplectique donnée, lorsque cette lagrangienne se trouve dans le lieu fixe d'une involution antisymplectique, laquelle est $J$-antiholomorphe et à condition que l'invariant que l'on a défini n'est pas nul. Nous souhaitons montrer ici qu'il est possible d'obtenir ces résultats pour une classe plus large de sous-variété lagrangiennes, en faisant intervenir la notion d'involutions antibirationnelles sur les variétés symplectiques.

\subsubsection{Involutions antibirationnelles des variétés symplectiques de dimension quatre}

Une involution $c_{X}$ de la variété symplectique de dimension quatre $(X, \omega)$ qui est définie en-dehors d'un nombre fini de points $x_{1}, \ldots, x_{k}$ de $X$ est dite antibirationnelle lorsqu'il existe un diagramme commutatif de la forme suivante :

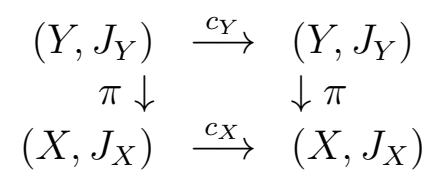

où $Y$ est une variété compacte de dimension quatre obtenue à partir de $X$ en réalisant un nombre fini d'éclatements topologiques au-dessus des points $x_{i}, i \in\{1, \ldots, k\}, J_{X}, J_{Y}$ sont des structures presque-complexes lisses et $c_{Y}$ une involution $J_{Y}$-antiholomorphe sur $Y$ toute entière. De plus, $J_{X}$ est supposée $\omega$-positive, $c_{X}$ est $J_{X}$-antiholomorphe sur son lieu de définition et $\pi$ est $\left(J_{Y}, J_{X}\right)$-holomorphe. 
Les involutions antibirationnelles classiques sur les surfaces compactes de Kähler fournissent des exemples de telles surfaces. Remarquons que pour tout $i \in\{1, \ldots, k\}, \pi^{-1}\left(x_{i}\right)$ est un arbre de sphères $J_{Y}$-holomorphes n'ayant que des points doubles transverses comme singularités.

Lemme 4.6 Supposons que pour tout $i \in\{1, \ldots, k\}$ et toute composante irréductible $C$ de l'arbre $\pi^{-1}\left(x_{i}\right), c_{Y}(C)$ ne soit pas contractée par $\pi$ sur $x_{1}, \ldots, x_{k}$. Alors, le diagramme ci-dessus est unique à équivalence près, une fois donnée $\left(X, \omega, c_{X}\right)$.

Soient $\left(X, \omega, J_{X}, c_{X}\right)$ satisfaisant les hypothèses du Lemme 4.6 et $\left(Y, J_{Y}, c_{Y}\right)$ la variété de dimension quatre associée. Soit $y$ l'ensemble fini $\left(\cup_{i=1}^{k} \pi^{-1}\left(x_{i}\right)\right) \cap c_{Y}\left(\cup_{i=1}^{k} \pi^{-1}\left(x_{i}\right)\right)$. L'involution antibirationnelle $c_{X}$ est dite simple lorsqu'elle satisfait les hypothèses du Lemme 4.6 et lorsque $\underline{y}$ se trouve en-dehors des points doubles de $\cup_{i=1}^{k} \pi^{-1}\left(x_{i}\right)$.

Lemme 4.7 Soit $c_{X}$ une involution antibirationnelle simple de $(X, \omega)$ et $\left(Y, c_{Y}\right)$ la variété de dimension quatre donnée par le Lemme 4.6. Alors, la deux-forme $\omega_{Y}=\pi^{*} \omega-\left(\pi \circ c_{Y}\right)^{*} \omega$ est fermée et non-dégénérée en tout point de $Y \backslash \underline{y}$. Elle est également non-dégénérée en tout point d'intersection transverse de $\left(\cup_{i=1}^{k} \pi^{-1}\left(x_{i}\right)\right) \cap c_{Y}\left(\cup_{i=1}^{k} \pi^{-1}\left(x_{i}\right)\right) \subset \underline{y}$.

Une telle deux-forme qui n'a qu'un nombre fini de noyaux de dimension deux sera dite quasi-symplectique. Remarquons qu'en particulier, lorsque l'intersection $\left(\cup_{i=1}^{k} \pi^{-1}\left(x_{i}\right)\right) \cap$ $c_{Y}\left(\cup_{i=1}^{k} \pi^{-1}\left(x_{i}\right)\right)$ est transverse, la deux-forme $\omega_{Y}$ est symplectique.

La structure presque-complexe $J_{Y}$ est $\omega_{Y}$-positive dans le sens que pour tous $y \in Y$ et $v \in T_{y} Y \backslash\{0\}$, soit $v$ et $J_{Y}(v)$ engendrent le noyau de $\left.\omega_{Y}\right|_{y}$, soit $\omega_{Y}\left(v, J_{Y}(v)\right)>0$. Notons $\mathcal{J}_{\omega_{Y}}$ l'espace des structures presque-complexes de classe $C^{l}$ qui sont $\omega_{Y}$-positives. Si $J \in \mathcal{J}_{\omega_{Y}}$, alors $\bar{c}_{Y}^{*}(J)=-d c_{Y} \circ J \circ d c_{Y}$ appartient également à $\mathcal{J}_{\omega_{Y}}$. Notons $\mathbb{R} \mathcal{J}_{\omega_{Y}}$ le lieu fixe de cette action de $\mathbb{Z} / 2 \mathbb{Z}$ sur $\mathcal{J}_{\omega_{Y}}$.

Lemme 4.8 Soient $c_{X}$ une involution antibirationnelle simple sur $(X, \omega)$ et $\left(Y, c_{Y}, \omega_{Y}\right)$ la variété de dimension quatre donnée par les Lemmes 4.6. 4.7. Il existe $J_{0} \in \mathbb{R} \mathcal{J}_{\omega_{Y}}$ tel que $\omega_{Y}\left(J_{0}, J_{0}\right)=\omega_{Y}$ et $g_{Y}=\omega_{Y}\left(., J_{0}\right)$ soit un deux-tenseur symétrique positif sur $Y$, défini en-dehors de $y$.

Pour tout voisinage $U$ de $\underline{y}$ et tout $J_{0} \in \mathbb{R} \mathcal{J}_{\omega_{Y}}$ donné par le Lemme 4.9 , notons $\mathcal{J}_{\omega_{Y}}^{U, J_{0}}$ (resp. $\mathbb{R} \mathcal{J}_{\omega_{Y}}^{U, J_{0}}$ ) le sous-espace $\overline{\operatorname{des}} J \in \mathcal{J}_{\omega_{Y}}$ (resp. $J \in \mathbb{R} \mathcal{J}_{\omega_{Y}}$ ) telles que $J=J_{0}$ sur $U$.

Lemme 4.9 Pour tous $U, J_{0}$, l'espace $\mathcal{J}_{\omega_{Y}}^{U, J_{0}}$ est une variété de Banach séparable non-vide et contractile. Le sous-espace $\mathbb{R} \mathcal{J}_{\omega_{Y}}^{U, J_{0}}$ en est une sous-variété de Banach séparable non-vide et contractiles.

Remarque 5 La deux-forme $\pi^{*} \omega$ est limite d'une suite de formes symplectiques sur $Y$ obtenues après un nombre fini d'éclatements de boules symplectiques dont les rayons convergent vers zéro. Par suite, la deux-forme $\omega_{Y}$ est limite d'une suite de formes symplectiques $\left(\omega_{Y}^{n}\right)_{n \in \mathbb{N}}$. Alors, $J \in \mathcal{J}_{\omega_{Y}}$ est $\omega_{Y}^{n}$-positif pour $n$ assez grand, principalement parce-que les noyaux de $\omega_{Y}$ deviennent symplectiques pour $\omega_{Y}^{n}$. 


\subsubsection{Invariants énumératifs des involutions antibirationnelles simples}

Soient $c_{X}$ une involution antibirationnelle simple sur $(X, \omega)$ et $x_{1}, \ldots, x_{k} \in X$ les points où elle n'est pas définie. Soit $\left(Y, \omega_{Y}, c_{Y}\right)$ la variété quasi-symplectique de dimension quatre associée, voir le Lemme 4.7. Soient $\pi$ la projection $Y \rightarrow X$ et $\underline{y}$ l'ensemble fini $\left(\cup_{i=1}^{k}\right.$ $\left.\pi^{-1}\left(x_{i}\right)\right) \cap c_{Y}\left(\cup_{i=1}^{k} \pi^{-1}\left(x_{i}\right)\right)$. Soit $\mathbb{R} Y$ le lieu fixe de $c_{Y}$, on étiquette ses composantes connexes $(\mathbb{R} Y)_{1}, \ldots,(\mathbb{R} Y)_{N}$. Remarquons que la courbe $\left(\cup_{i=1}^{k} \pi^{-1}\left(x_{i}\right)\right) \cup c_{Y}\left(\cup_{i=1}^{k} \pi^{-1}\left(x_{i}\right)\right)$ n'intersecte $\mathbb{R} Y$ qu'en un nombre fini de points, de sorte qu'elle ne déconnecte aucune des courbes $(\mathbb{R} Y)_{i}, i \in\{1, \ldots, N\}$. Soient $d_{Y} \in H_{2}(Y ; \mathbb{Z})$ tel que $\left(c_{Y}\right)_{*} d_{Y}=-d_{Y}, c_{1}(Y) d_{Y}>0$ et $y=\left(y_{1}, \ldots y_{c_{1}(Y) d_{Y}-1}\right)$ une configuration réelle de $c_{1}(Y) d_{Y}-1$ points distinct de $Y \backslash$ $\left(\cup_{i=1}^{k} \pi^{-1}\left(x_{i}\right) \cup c_{Y}\left(\cup_{i=1}^{k} \pi^{-1}\left(x_{i}\right)\right)\right)$. Pour tout $i \in\{1, \ldots, N\}$, notons $r_{i}=\#\left(y \cap(\mathbb{R} Y)_{i}\right)$ puis $r=\left(r_{1}, \ldots, r_{N}\right)$. Soient $U$, voisinage de $\underline{y}$ et $J_{0} \in \mathbb{R} \mathcal{J}_{\omega_{Y}}$ donnés par le Lemme 4.9. Alors, dès que $U$ est suffisamment petit, pour tout $J \in \mathbb{R}_{\mathcal{J}_{Y}}^{U, J_{0}}$ générique, il n'y a qu'un nombre fini de courbes $J$-holomorphes rationnelles réelles homologue à $d_{Y}$ dans $Y$ qui contiennent $y$. Ces courbes sont toutes irréductibles, immergées et n'ont que des points doubles transverses comme singularités. Le nombre total de leurs points doubles vaut $\delta_{Y}=\frac{1}{2}\left(d_{Y}^{2}-c_{1}(Y) d_{Y}+2\right)$. Pour tout entier $m$ compris entre 0 et $\delta_{Y}$, notons $n_{d}(m)$ le nombre de ces courbe qui sont de masse $m$. On pose alors

$$
\chi_{r}^{d_{Y}}\left(y, J, U, J_{0}\right)=\sum_{m=0}^{\delta_{Y}}(-1)^{m} n_{d}(m) .
$$

Théorème 4.10 L'entier $\chi_{r}^{d_{Y}}\left(y, J, U, J_{0}\right)$ ne dépend pas des choix de $y, J, U$ et $J_{0}$.

Remarquons que l'entier $\chi_{r}^{d_{Y}}$ fourni par le Théorème 4.10 est un invariant par déformation du triplet $\left(X, \omega, c_{X}\right)$, puisque le triplet $\left(Y, \omega_{Y}, c_{Y}\right)$ lui est canoniquement associé.

\subsubsection{Exemple : les tores isotopes au tore de Clifford}

Soient $a, b \in \mathbb{R}_{+}^{*}$ et $\mathbb{T}_{a, b} \subset \mathbb{C} P^{2}$ le tore lagrangien défini par les équations $|x|=a$, $|y|=b$ dans les coordonnées affines $(x, y) \in(\mathbb{C})^{2} \subset \mathbb{C} P^{2}$. Ce tore est le lieu fixe de l'involution antibirationnelle de Cremona $c^{a, b}:(x, y, z) \in \mathbb{C} P^{2} \backslash\{(1,0,0),(0,1,0),(0,0,1)\} \mapsto$ $\left(a^{2} \overline{y z}, b^{2} \overline{x z}, \overline{x y}\right) \in \mathbb{C} P^{2}$. Cette involution antibirationnelle $c^{a, b}, a, b \in \mathbb{R}_{+}^{*}$, est simple. En effet, soit $Y$ le plan projectif éclaté aux trois points $(1,0,0),(0,1,0),(0,0,1)$ et $\pi: Y \rightarrow \mathbb{C} P^{2}$ la projection associée. L'involution $c^{a, b}$ se relève en une involution antiholomorphe $c_{Y}^{a, b}$ définie partout, soit une structure réelle. Cette dernière envoie les trois diviseurs exceptionnels sur les transformées strictes des côtés du triangle $(1,0,0),(0,1,0),(0,0,1)$, d'où la simplicité de $c^{a, b}$. Ainsi, le Théorème 4.10 s'applique et fournit des invariants $\chi_{r}^{d_{Y}}$ par déformation du triplet $\left(\mathbb{C} P^{2}, \omega, c^{a, b}\right)$. Le deuxième groupe d'homologie de $Y$ est engendré par une droite générique et les diviseurs exceptionnels $E_{1}, \ldots E_{3}$ de nos éclatements. La classe d'homologie $d_{Y}$ de nos courbes rationnelles réelles de $Y$ est déterminée par quatre entiers $d, d_{1}, \ldots, d_{3}$ satisfaisant la relation $d=d_{1}+d_{2}+d_{3}$. Si l'on contracte $E_{1}, \ldots E_{3}$, ces courbes se contractent sur des courbes rationnelles de degré $d$ du plan qui ont un point de 
multiplicité $d_{1}, d_{2}, d_{3}$ en $(1,0,0),(0,1,0),(0,0,1)$ respectivement. Ces courbes rationnelles immergées ont en outre la propriété de rencontrer le tore $\mathbb{T}_{a, b}$ en une collection de points isolés et en un cercle immergé, elles consistent en fait en une paire de disques $J$-holomorphes qui reposent sur $\mathbb{T}_{a, b}$ et sont échangés par $c^{a, b}$. Si l'on contracte plutôt un diviseur exceptionnel, disons $E_{3}$, ainsi que son image sous $c_{Y}^{a, b}$, alors on obtient des courbes rationnelles de bidegré $\left(d_{1}+d_{3}, d_{2}+d_{3}\right)$ sur l'hyperboloïde quadrique $\left(\mathbb{C} P^{1} \times \mathbb{C} P^{1}\right.$, conj $\times$ conj), qui ont une paire de points de multiplicité $d_{3}$ en deux points complexes conjugués, à savoir les points où $E_{3}$ et son image se contractent. Lorsque $d_{3}=0$ ou 1 , cet invariant $\chi_{r}^{d_{Y}}$ vaut l'invariant correspondant dans l'hyperboloïde quadrique $\left(\mathbb{C} P^{1} \times \mathbb{C} P^{1}\right.$, conj $\times$ conj $)$, à savoir $\chi_{r}^{\left(d_{1}, d_{2}\right)}$ et $\chi_{r}^{\left(d_{1}+1, d_{2}+1\right)}$ respectivement. Des estimations de ces derniers se trouvent dans [12].

Corollaire 11 Soient $r, s, d \in \mathbb{N}$ tels que $r+2 s=2 d-1$ et supposons donnée une collection de $r$ points distincts dans $\mathbb{T}_{a, b} \subset \mathbb{C} P^{2}, a, b \in \mathbb{R}_{+}^{*}$ ainsi qu'une collection de s paires distinctes de points dans $\mathbb{C} P^{2} \backslash\left(\mathbb{T}_{a, b} \cup\{(1,0,0),(0,1,0),(0,0,1)\}\right)$ échangées par l'involution antibirationnelle $c^{a, b}$. Alors, pour tous $d_{1}, d_{2}, d_{3} \in \mathbb{N}$ tels que $d=d_{1}+d_{2}+d_{3}$, il y a au moins $\left|\chi_{r}^{d_{Y}}\right|$ paires de disques $J_{X}$-holomorphes reposant sur $\mathbb{T}_{a, b}$, échangés par $c^{a, b}$, passant par les $r$ points donnés et intersectant chacune des s paires de points complexes conjugués, dès lors que $J_{X}$ se relève en une structure $J_{Y}$ appartenant à l'un des $\mathbb{R} \mathcal{J}_{\omega_{Y}}^{U, J_{0}}$ donné par le Lemme 4.9. La réunion de ces deux disques dans chacune de ces paires forme une courbe rationnelle plane de degré $d$ ayant un point de multiplicité $d_{1}, d_{2}, d_{3}$ en $(1,0,0),(0,1,0),(0,0,1)$ respectivement.

Remarquons que des invariants énumératifs portant sur des disques à bords dans le tore de Clifford ont été obtenus par P. Biran et O. Cornea [2]. Les disques holomorphes à bords dans le tores de Clifford ont par ailleurs été étudiés par C.-H. Cho dans sa thèse en termes de produits de Blaschke. En ce qui concerne nos résultats présentés dans ce paragraphe 4.2. il reste à s'affranchir de la notion de simplicité (des involutions antibirationnelles).

\section{Références}

[1] A. Arroyo, E. Brugallé, and L. López De Medrano. Recursive formulas for Welschinger invariants. Prépublication math.arXiv :0809.1541, 2008.

[2] P. Biran and O. Cornea. A Lagrangian quantum homology. In New perspectives and challenges in symplectic field theory, volume 49 of CRM Proc. Lecture Notes, pages 1-44. Amer. Math. Soc., Providence, RI, 2009.

[3] F. Bourgeois. A Morse-Bott approach to Contact Homology. Ph.D dissertation, Stanford University, 2002.

[4] E. Brugallé and G. Mikhalkin. Enumeration of curves via floor diagrams. C. R. Math. Acad. Sci. Paris, 345(6) :329-334, 2007.

[5] C.-H. Cho. Counting real $J$-holomorphic discs and spheres in dimension four and six. J. Korean Math. Soc., 45(5) :1427-1442, 2008. 
[6] K. Fukaya, Y.-G. Oh, H. Ohta, and K. Ono. Lagrangian intersection Floer theory : anomaly and obstruction. Part I, volume 46 of AMS/IP Studies in Advanced Mathematics. American Mathematical Society, Providence, RI, 2009.

[7] K. Fukaya, Y.-G. Oh, H. Ohta, and K. Ono. Lagrangian intersection Floer theory : anomaly and obstruction. Part II, volume 46 of AMS/IP Studies in Advanced Mathematics. American Mathematical Society, Providence, RI, 2009.

[8] A. Gathmann and H. Markwig. The Caporaso-Harris formula and plane relative Gromov-Witten invariants in tropical geometry. Math. Ann., 338(4) :845-868, 2007.

[9] M. Gromov. Pseudoholomorphic curves in symplectic manifolds. Invent. Math., 82(2) :307-347, 1985.

[10] A. Grothendieck. Sur la classification des fibrés holomorphes sur la sphère de Riemann. Amer. J. Math., 79 :121-138, 1957.

[11] H. Hofer, K. Wysocki, and E. Zehnder. Properties of pseudoholomorphic curves in symplectisations. I. Asymptotics. Ann. Inst. H. Poincaré Anal. Non Linéaire, 13(3) :337-379, 1996.

[12] I. Itenberg, V. Kharlamov, and E. Shustin. Welschinger invariant and enumeration of real rational curves. Int. Math. Res. Not., (49) :2639-2653, 2003.

[13] I. Itenberg, V. Kharlamov, and E. Shustin. A Caporaso-Harris type formula for Welschinger invariants of real toric del Pezzo surfaces. Comment. Math. Helv., 84(1) :87126, 2009.

[14] I. V. Itenberg, V. M. Kharlamov, and E. I. Shustin. Logarithmic equivalence of the Welschinger and the Gromov-Witten invariants. Uspekhi Mat. Nauk, 59(6(360)) :85110, 2004.

[15] A. I. Degtyarev and V. M. Kharlamov. Topological properties of real algebraic varieties : Rokhlin's way. Uspekhi Mat. Nauk, 55(4(334)) :129-212, 2000.

[16] G. Mikhalkin. Enumerative tropical algebraic geometry in $\mathbb{R}^{2}$. J. Amer. Math. Soc., 18(2) :313-377 (electronic), 2005.

[17] R. Pandharipande, J. Solomon, and J. Walcher. Disk enumeration on the quintic 3-fold. J. Amer. Math. Soc., 21(4) :1169-1209, 2008.

[18] N. Puignau. Première classe de Stiefel-Whitney des espaces d'applications stables réelles en genre zéro vers une surface convexe. J. Inst. Math. Jussieu, 8(2) :383-414, 2009.

[19] F. Ronga, A. Tognoli, and T. Vust. The number of conics tangent to five given conics : the real case. Rev. Mat. Univ. Complut. Madrid, 10(2) :391-421, 1997.

[20] E. Shustin. A tropical calculation of the Welschinger invariants of real toric del Pezzo surfaces. J. Algebraic Geom., 15(2) :285-322, 2006.

[21] E. Shustin. Welschinger invariants of toric del Pezzo surfaces with nonstandard real structures. Tr. Mat. Inst. Steklova, 258(Anal. i Osob. Ch. 1) :227-255, 2007. 
[22] J. P. Solomon. Intersection theory on the moduli space of holomorphic curves with Lagrangian boundary conditions. Prépublication math.SG/0606429, 2006.

[23] J.-Y. Welschinger. Invariants of real rational symplectic 4-manifolds and lower bounds in real enumerative geometry. C. R. Math. Acad. Sci. Paris, 336(4) :341-344, 2003.

[24] J.-Y. Welschinger. Enumerative invariants of strongly semipositive real symplectic six-manifolds. Prépublication math.AG/0509121, 2005.

[25] J.-Y. Welschinger. Invariants of real symplectic 4-manifolds and lower bounds in real enumerative geometry. Invent. Math., 162(1) :195-234, 2005.

[26] J.-Y. Welschinger. Spinor states of real rational curves in real algebraic convex 3manifolds and enumerative invariants. Duke Math. J., 127(1) :89-121, 2005.

[27] J.-Y. Welschinger. Invariants of real symplectic four-manifolds out of reducible and cuspidal curves. Bull. Soc. Math. France, 134(2) :287-325, 2006.

[28] J.-Y. Welschinger. Towards relative invariants of real symplectic four-manifolds. Geom. Funct. Anal., 16(5) :1157-1182, 2006.

[29] J.-Y. Welschinger. Invariant count of holomorphic disks in the cotangent bundles of the two-sphere and real projective plane. C. R. Math. Acad. Sci. Paris, 344(5) :313-316, 2007.

[30] J.-Y. Welschinger. Optimalité, congruences et calculs d'invariants des variétés symplectiques réelles de dimension quatre. Prépublication math.SG/0707.4317, 2007.

[31] J.-Y. Welschinger. Open strings, Lagrangian conductors and Floer functor. Prépublication math.arXiv :0812.0276, 2008.

Université de Lyon; CNRS ; Université Lyon 1; Institut Camille Jordan 